\title{
DIFFUSE $\gamma$-RAY EMISSION FROM MISALIGNED ACTIVE GALACTIC NUCLEI
}

\author{
M. Di Mauro ${ }^{1}$, F. Calore 2 , F. Donato ${ }^{1}$, M. Ajello ${ }^{3}$, and L. Latronico ${ }^{4}$ \\ ${ }^{1}$ Physics Department, Torino University, and Istituto Nazionale di Fisica Nucleare, Sezione di Torino, \\ via Giuria 1, I-10125 Torino, Italy; donato@to.infn.it \\ 2 II. Institute for Theoretical Physics, University of Hamburg, Luruper Chaussee 149, D-22761 Hamburg, Germany \\ ${ }^{3}$ Space Sciences Laboratory, University of California, Berkeley, CA 94720, USA \\ ${ }^{4}$ Istituto Nazionale di Fisica Nucleare, Sezione di Torino, via Giuria 1, I-10125 Torino, Italy \\ Received 2013 April 3; accepted 2013 November 17; published 2013 December 20
}

\begin{abstract}
Active galactic nuclei (AGNs) with jets seen at small viewing angles are the most luminous and abundant objects in the $\gamma$-ray sky. AGNs with jets misaligned along the line of sight appear fainter in the sky but are more numerous than the brighter blazars. We calculate the diffuse $\gamma$-ray emission due to the population of misaligned AGNs (MAGNs) unresolved by the Large Area Telescope (LAT) on the Fermi Gamma-ray Space Telescope (Fermi). A correlation between the $\gamma$-ray luminosity and the radio-core luminosity is established and demonstrated to be physical by statistical tests, as well as compatible with upper limits based on Fermi-LAT data for a large sample of radio-loud MAGNs. We constrain the derived $\gamma$-ray luminosity function by means of the source-count distribution of the radio galaxies detected by the Fermi-LAT. We finally calculate the diffuse $\gamma$-ray flux due to the whole MAGN population. Our results demonstrate that MAGNs can contribute from $10 \%$ up to nearly the entire measured isotropic gamma-ray background. We evaluate a theoretical uncertainty on the flux of almost an order of magnitude.
\end{abstract}

Key words: diffuse radiation - galaxies: active - galaxies: luminosity function, mass function

Online-only material: color figures

\section{INTRODUCTION}

The Fermi Large Area Telescope (LAT) has measured the isotropic gamma-ray background (IGRB) with very good accuracy from $200 \mathrm{MeV}$ to $100 \mathrm{GeV}$ (Abdo et al. 2010d). Increased statistics from additional LAT data are expected to allow the IGRB to be measured over an even broader energy range. The nature of the IGRB is still an open problem in astrophysics. Blazars and star-forming galaxies contribute $20 \%-30 \%$ of the IGRB (Abdo et al. 2010e; Ackermann et al. 2012a) and with a compatible spectral slope. Blazars are active galactic nuclei (AGNs) whose jets are oriented along their lines of sight (LOSs). Their luminosity is quite high due to Doppler-boosting effects. For the same reason, AGNs with axes misaligned with respect to the LOS (hereafter misaligned AGNs (MAGNs)) have weaker luminosities but are expected to be more numerous by a factor of $\sim 2 \Gamma_{L}^{2}$ (where $\Gamma_{L}$ is the Lorentz factor, Urry \& Padovani 1995). About $10 \%$ of the observed AGNs are radio-loud. According to the unified model, AGNs are classified as a function of their jet orientation with respect to the LOS. A jet misalignment of about $14^{\circ}$ distinguishes blazars from non-blazars, i.e., misaligned objects (Urry \& Padovani 1995). In the unified model, radio galaxies (RGs) are those objects that, on average, have their jets pointing at $>44^{\circ}$ from our LOS, while MAGNs below this angle and above about $14^{\circ}$ are generally identified with radio quasars (Barthel 1989). RGs are classified in turn into two categories based upon their radio morphology (Fanaroff \& Riley 1974). The first class of objects, named Fanaroff-Riley Type I (FRI), is preferentially found in rich clusters, hosted by weak-lined galaxies, and has a low luminosity radio emission (which peaks near the center of the AGN and shows two-sided, jets-dominated emission). Fanaroff-Riley Type II (FRII) galaxies present a high-luminosity radio emission dominated by the lobes, while jets and core (when detected) are faint. The hot spots, generally not present in FRIs, are usually detected at the ends of the lobes.
The threshold in luminosity for discriminating between FRIs and FRIIs is about $10^{25} \mathrm{~W} \mathrm{~Hz}^{-1} \mathrm{sr}^{-1}$ at $178 \mathrm{MHz}$ (Fanaroff \& Riley 1974). FRIs and FRIIs are considered the parent populations of BL Lacertae objects (BL Lacs) and flat-spectrum radio quasars (FSRQs), respectively.

A recent analysis finds that the Faint Images of the Radio Sky at Twenty centimeters (FIRST) radio sources undetected by the Fermi LAT may explain about one-half of the IGRB (Zhou $\&$ Wang 2013). The contribution of unresolved blazars to the IGRB has been shown to be non-negligible (Stecker et al. 1993; Padovani et al. 1993; Salamon \& Stecker 1994; Abazajian et al. 2011; Abdo et al. 2010e) and able to explain at least $10 \%$ of the measured IGRB at high latitudes (Abdo et al. 2010d). Given the large numbers of known radio-loud MAGNs, and in analogy with blazars, we estimate in this work how the faint but numerous unresolved MAGN population may contribute to the IGRB at a non-negligible level (Stawarz et al. 2006; Bhattacharya et al. 2009; Inoue 2011). Using the first year Fermi-LAT catalog, Inoue (2011) evaluated the contribution of MAGNs to be between $10 \%$ and $63 \%$ of the IGRB. We investigate the absolute level of the MAGN $\gamma$-ray flux and quantify the possible uncertainties of our prediction. The main original points of our analysis include: (1) the derivation of a $\gamma$-ray-radio-core luminosity correlation for the MAGNs detected by Fermi-LAT; (2) a check of this correlation against upper limits (ULs) from tens of radio loud MAGNs undetected in $\gamma$-rays; (3) statistical tests that verify that the evaluation of the radio-core- $\gamma$-ray luminosity correlation is not spurious; (4) the computation of the $\gamma$-ray luminosity function (GLF) from the core radio one; (5) evaluation of the uncertainties affecting the $\gamma$-ray flux predicted from the unresolved MAGN population.

In Section 2, we derive the correlation between radiocore $\left(L_{r, \text { core }}\right)$ and $\gamma$-ray luminosities $\left(L_{\gamma}\right)$ from a sample of 12 MAGNs detected by Fermi-LAT. The robustness of the $L_{\gamma}-L_{r \text {,core }}$ correlation is tested in Section 3 by computing the $95 \%$ confidence level (CL) ULs on the $\gamma$-ray flux of a sample 
of radio-loud MAGNs with 4 yr of Fermi data. In Section 4, two statistical tests are performed on the core radio and $\gamma$-ray data in order to exclude spurious effects in the correlation between luminosities. By assuming the relation found between $L_{\gamma}$ and $L_{r \text {,core }}$, in Section 5 we model the $\gamma$-ray luminosity function from the radio luminosity function (RLF). We discuss the consistency of the models in Section 6, where we compare our predictions of the source-count distribution and compare them to the FermiLAT data. Our findings for the contribution of an unresolved population of MAGNs to the IGRB are presented in Section 7 together with the evaluation of the relevant uncertainties. Finally, we draw our conclusions in Section 8.

Throughout the paper we adopt a standard $\Lambda$ CDM cosmology with parameters $H_{0}=70 \mathrm{~km} \mathrm{~s}^{-1} \mathrm{Mpc}^{-1}, \Omega_{M}=0.27$, and $\Omega_{\Lambda}=0.73$.

\section{THE CORRELATION BETWEEN $\gamma$-RAY AND RADIO LUMINOSITY}

The calculation of the diffuse emission from unresolved (i.e., not detected by the Fermi-LAT) MAGNs relies on the GLF for that specific population. The physical processes underlying the emission of $\gamma$ rays in RGs are not firmly established. However, by analogy with blazars-being the same objects with off-line axes-it is commonly assumed that MAGNs experience the same emission processes (Grandi 2012). It is believed that the bulk of the radiation is generated via synchrotron self-Compton (SSC) scatterings, where the seed photons are provided by synchrotron emission by the same electron population (Maraschi et al. 1992). External inverse Compton (EC) scattering off photons external to the jet is not excluded (Dermer \& Schlickeiser 1993). Dedicated studies of M87 (Abdo et al. 2009a), Cen A (Abdo et al. 2010a), and NGC 1275 (Abdo et al. 2009c) show that the SSC process successfully fits the observed emission over a wide photon energy range, even if other mechanisms have been explored (Kusenko \& Voloshin 2012).

The contribution of kiloparsec-scale jets and radio lobes to the IGRB is less than 10\% (Stawarz et al. 2006; Massaro \& Ajello 2011). Ultrarelativistic electrons in the lobes emit synchrotron radiation in the radio band and are able to upscatter low-energy photons via inverse Compton (IC) scattering to high energies, provided a high enough electron density is available. The dominant contribution is expected to be from cosmic microwave background (CMB) photons. The IC/CMB scattered emission in the lobes of distant galaxies is generally well observed in the X-ray band. Extended $\gamma$-ray emission that is spatially coincident with radio lobes has been detected from Cen A (Abdo et al. 2010b). Such emission, if interpreted in terms of IC scattering of electrons with ambient photons, requires highenergy electrons in the lobes, but it is unclear how common this is in other RGs. In what follows, we assume the $\gamma$-ray radiation originates in the central region of the source, as is predicted from both SSC and EC scenarios.

The FRI and FRII galaxies show a strong emission in a wide radio band, spanning from hundreds of $\mathrm{MHz}$ up to tens of $\mathrm{GHz}$. These photons are ascribed to the synchrotron emission of highly relativistic electrons moving in the entire region of the source. The total radio flux has been measured for hundreds of FRI and FRII galaxies. For a number of these galaxies, the emission from the central unresolved region of an arcsecond scale, often referred to as the core, is detected as well. In the first and second catalogs of the LAT AGN sources (Abdo et al. 2010c; Ackermann et al. 2011b), Fermi-LAT has reported the detection of 15 MAGNs, which can be classified into 10 FRI and 5 FRII galaxies (although with some caveats, see below). Abdo et al. (2010c) report on the observation of $3 \mathrm{C} 78$, PKS 0625-35, 3C 207, 3C 274, Cen A, NGC 6251, 3C 380, 3C 120, 3C 111, 3C 84, and PKS 0943-76, while Cen B, Fornax A, and IC 310 have been reported in the second LAT catalog (2FGL; Nolan et al. 2012a, 2012b; for Cen B, see also Katsuta et al. 2013) and a Pictor A identification has been discussed in Brown \& Adams (2012).

In the absence of predictions for the GLF, we follow a phenomenological approach to relate the $\gamma$-ray luminosity to the radio luminosity, as it is commonly done in the literature for source populations and notably for RGs with the 1FGL data set (Inoue 2011). We explore here for the first time the correlation between the core radio and the $\gamma$-ray luminosity, and adopt an RLF from the literature. The latter is phenomenologically much better established, given that the number of detected MAGNs in the radio frequencies should be high. A possible correlation between radio and $\gamma$-ray luminosities has been proposed for blazars using the Energetic Gamma Ray Experiment Telescope (EGRET) data (Padovani et al. 1993; Stecker et al. 1993; Salamon \& Stecker 1994; Dondi \& Ghisellini 1995; Narumoto $\&$ Totani 2006). Recently, the connection between radio and $\gamma$-ray fluxes has been explored for both the FSRQs and BL Lacs detected by Fermi-LAT during its first year of operation (Ackermann et al. 2011a; Ghirlanda et al. 2011). On a similar basis, the relation between radio emission and $\gamma$-ray data has been studied for three FRI galaxies observed by EGRET (Ghisellini et al. 2005), as well as for FRI and FRII galaxies with 15 months of data taken with the Fermi-LAT (Inoue 2011; Abdo et al. 2010c). Variability studies for FRI galaxies support the hypothesis of the compactness of the $\gamma$-ray source (Abdo et al. 2010c; Grandi et al. 2012a), even if a non-negligible $\gamma$-ray counterpart in radio lobes has been observed in Cen A (Abdo et al. 2010b). The situation for the FRII population is less definite. A recent Fermi-LAT analysis of the FRII 3C 111 galaxy (Grandi et al. 2012b), together with a multi-frequency campaign conducted in the same period, localizes the $\mathrm{GeV}$ photons from 3C 111 in a compact, central region associated with the radio core.

The main radio and $\gamma$ parameters of all the MAGNs observed by Fermi-LAT are reported in Table 1 . The radio data were chosen to be the closest in time to the data from Fermi-LAT. Whenever a significant variability was found, we selected radio data as contemporary as possible to the $\gamma$-ray observations. Radio data were taken with the Very Large Array for all the objects except NGC 6251, which was measured with the very long baseline interferometer (VLBI). The linear size scales explored by the instruments depend on the redshift of the sources. In our sample, they vary from about $0.01 \mathrm{kpc}$ to a few $\mathrm{kpc}^{5}$ except for NGC 6251 and 3C 380. Data for 3C 380 are taken from Effelsberg observations. However, this source shows a compact steep-spectrum radio morphology, and the radio flux from the central region is close to both the total emission and the flux measured within a few arcseconds scale. ${ }^{6}$ For $3 \mathrm{C} 84$, the variability is very pronounced and we have therefore excluded

\footnotetext{
5 3C 78: 2 kpc, 3C 274: 40 pc, Cen A: 20 pc, NGC 6251: 2 pc, Cen B: $0.5 \mathrm{kpc}$, For A: $0.1 \mathrm{kpc}, 3 \mathrm{C}$ 120: $2.3 \mathrm{kpc}$, PKS 0625-35: 10 pc, Pictor A: $6.9 \mathrm{kpc}, 3 \mathrm{C}$ 111: $0.38 \mathrm{kpc}, 3 \mathrm{C}$ 207: $2.8 \mathrm{kpc}, 3 \mathrm{C} 380: 73 \mathrm{kpc}$. The radio measurements of some objects (e.g., 3C 207, 3C 280) might be contaminated by the extended jet emission. However, the uncertainty introduced by this likely contamination is one of the uncertainties contributing to the scatter of Figure 1 and, as such, is factored into our analysis.

6 http://3crr.extragalactic.info/cgi/database
} 
Main Radio and $\gamma$-ray Properties of the MAGNs Observed by Fermi-LAT

\begin{tabular}{|c|c|c|c|c|c|c|c|c|}
\hline MAGN (FRI, FRII) & $z$ & $\begin{array}{l}b \\
\left({ }^{\circ}\right)\end{array}$ & $\alpha_{\text {core }}\left(\alpha_{\text {tot }}\right)$ & $S_{\mathrm{core}}^{5 \mathrm{GHz}}(\mathrm{Jy})\left(S_{\mathrm{tot}}^{5 \mathrm{GHz}}\right)(\mathrm{Jy})$ & $\Gamma$ & $\begin{array}{c}F_{\gamma} \\
\left(10^{-9} \text { photons } \mathrm{cm}^{-2} \mathrm{~s}^{-1}\right)\end{array}$ & $\begin{array}{c}L_{r, \text { cor }^{-1}}^{5 \mathrm{GHz}} \\
\left(\mathrm{erg} \mathrm{s}^{-1}\right)\end{array}$ & $\begin{array}{c}L_{\gamma} \\
\left(\mathrm{erg} \mathrm{s}^{-1}\right)\end{array}$ \\
\hline 3C 78/NGC 1218(I) & 0.0287 & -44.6 & $0\left(0.64^{1}\right)$ & $0.964 \pm 0.164^{1}\left(3.40 \pm 0.11^{2}\right)$ & $1.95 \pm 0.14$ & $4.7 \pm 1.8$ & $(8.8 \pm 1.4) \times 10^{40}$ & $(1.11 \pm 0.54) \times 10^{43}$ \\
\hline $3 \mathrm{C} 274 / \mathrm{M} 87(\mathrm{I})$ & 0.0038 & 74.5 & $0\left(0.79^{8}\right)$ & $3.0971 \pm 0.0300^{7}\left(71.566 \pm 0.993^{9}\right)$ & $2.17 \pm 0.07$ & $25.8 \pm 3.5$ & $(4.90 \pm 0.05) \times 10^{39}$ & $(6.2 \pm 1.1) \times 10^{41}$ \\
\hline Cen A(I) & 0.0009 & 19.4 & $0.30^{10}\left(0.70^{10}\right)$ & $6.984 \pm 0.210^{11}\left(62.837 \pm 0.099^{12}\right)$ & $2.76 \pm 0.05$ & $175 \pm 10$ & $(6.19 \pm 0.19) \times 10^{38}$ & $(1.14 \pm 0.09) \times 10^{41}$ \\
\hline NGC 6251(I) & 0.0247 & 31.2 & $0\left(0.72^{9}\right)$ & $0.38 \pm 0.04^{13}\left(0.510 \pm 0.050^{13 a}\right)$ & $2.20 \pm 0.07$ & $18.2 \pm 2.6$ & $(2.57 \pm 0.27) \times 10^{40}$ & $(1.82 \pm 0.41) \times 10^{43}$ \\
\hline Cen B(I) & 0.0129 & 1.68 & $0\left(0.13^{16}\right)$ & $2.730^{15}\left(6.58 \pm 1.04^{16}\right)$ & $2.33 \pm 0.12$ & $39.3 \pm 11.4$ & $5.02 \times 10^{40}$ & $(8.6 \pm 3.2) \times 10^{42}$ \\
\hline For A(I) & 0.00587 & -56.7 & $0.50^{17}\left(0.52^{1}\right)$ & $0.051^{17}\left(72^{1}\right)$ & $2.16 \pm 0.15$ & $7.7 \pm 2.4$ & $1.93 \times 10^{38}$ & $(4.6 \pm 2.2) \times 10^{41}$ \\
\hline $3 \mathrm{C} 120(\mathrm{I})$ & 0.0330 & -27.4 & $0\left(0.44^{18}\right)$ & $3.458 \pm 0.588^{1}\left(8.60 \pm 1.46^{1}\right)$ & $2.71 \pm 0.35$ & $29 \pm 17$ & $(4.20 \pm 0.71) \times 10^{41}$ & $(2.9 \pm 1.6) \times 10^{43}$ \\
\hline PKS $0625-35(I)^{b}$ & 0.0546 & -20.0 & $0\left(0.65^{3}\right)$ & $0.600 \pm 0.030^{3}\left(2.25 \pm 0.09^{4}\right)$ & $1.93 \pm 0.09$ & $12.9 \pm 2.6$ & $(2.02 \pm 0.10) \times 10^{41}$ & $(1.21 \pm 0.43) \times 10^{44}$ \\
\hline Pictor A(II) & 0.0351 & -34.6 & $0\left(1.07^{1}\right)$ & $1.15 \pm 0.05^{19}\left(15.45 \pm 0.47^{4}\right)$ & $2.93 \pm 0.03$ & $21.9 \pm 3.6$ & $(1.58 \pm 0.07) \times 10^{41}$ & $(2.13 \pm 0.46) \times 10^{43}$ \\
\hline $3 \mathrm{C} 111(\mathrm{II})$ & 0.0485 & -8.61 & $-0.20^{\mathrm{b}}\left(0.73^{5}\right)$ & $1.14^{20}\left(6.637 \pm 0.996^{18}\right)$ & $2.54 \pm 0.19$ & $40 \pm 8$ & $2.98 \times 10^{41}$ & $(1.01 \pm 0.38) \times 10^{44}$ \\
\hline $3 \mathrm{C} 207(\mathrm{II})^{\mathrm{c}}$ & 0.681 & 30.1 & $0\left(0.90^{5}\right)$ & $0.5391 \pm 0.0030^{6}\left(1.35 \pm 0.04^{4}\right)$ & $2.36 \pm 0.11$ & $17.3 \pm 3.3$ & $(3.32 \pm 0.02) \times 10^{43}$ & $(2.41 \pm 0.61) \times 10^{46}$ \\
\hline $3 \mathrm{C} 380(\mathrm{II})^{\mathrm{c}}$ & 0.692 & 23.5 & $0\left(0.71^{9}\right)$ & $5.073 \pm 0.105^{14}\left(7.45 \pm 0.37^{4}\right)$ & $2.34 \pm 0.07$ & $30.3 \pm 3.7$ & $(3.12 \pm 0.07) \times 10^{44}$ & $(4.44 \pm 0.73) \times 10^{46}$ \\
\hline IC 310(I) & 0.0189 & -13.7 & n.a. $\left(0.75^{23}\right)$ & n.a. $\left(0.258 \pm 0.031^{24}\right)$ & $2.10 \pm 0.19$ & $11.1 \pm 6.2$ & $\ldots$ & $(7.9 \pm 4.9) \times 10^{42}$ \\
\hline $3 \mathrm{C} 84 /$ NGC $1275(\mathrm{I})$ & 0.0176 & -13.2 & $\left(0.78^{5}\right)$ & High variability & $2.00 \pm 0.02$ & $175 \pm 8$ & $\ldots$ & $(1.22 \pm 0.07) \times 10^{44}$ \\
\hline PKS 0943-76(II) & 0.270 & -17.2 & n.a. & Upper limits $\left(0.757^{22}\right)$ & $2.44 \pm 0.14$ & $19.5 \pm 5.1$ & $\ldots$ & $(2.47 \pm 0.71) \times 10^{45}$ \\
\hline
\end{tabular}

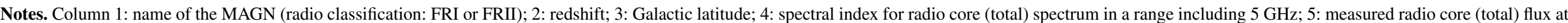

$5 \mathrm{GHz}$; 6: photon index for $\gamma$-ray spectrum between $100 \mathrm{MeV}$ and $100 \mathrm{GeV}$; $: \gamma$-ray flux above $100 \mathrm{MeV}$; 8: radio core luminosity at 5 GHz; 9: $\gamma$-ray luminosity.

${ }^{a}$ VLBI core+jet data, used in our analysis.

${ }^{\mathrm{b}}$ Our interpolation.

${ }^{c}$ Non-standard.

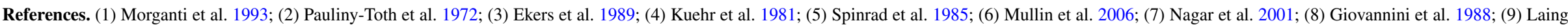

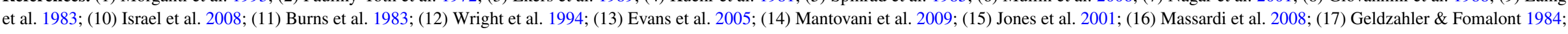
(18) Becker et al. 1991; (19) Perley et al. 1997; (20) Linfield \& Perley 1984; (21) Kadler et al. 2012; (22) Burgess \& Hunstead 2006; (23) Kadler et al. 2012 ; (24) Gregory \& Condon 1991. 
it from our correlation analysis. IC 310 lacks measurements of the core at $5 \mathrm{GHz}$, and the total radio flux is very faint. For PKS 0943-76, only ULs for the core are given. For these reasons, these two galaxies are listed but not considered in our analysis. ${ }^{7}$ The photon index, $\Gamma$, valid between 0.1 and $10 \mathrm{GeV}$, and the Fermi-LAT flux integrated for $E_{\gamma}>0.1 \mathrm{GeV}$ have been taken from Abdo et al. (2010c) for 3C 78, 3C 111, and 3C 120, and from Nolan et al. (2012b) for the remaining objects. From Table 1 , the mean photon index, $\Gamma$, is 2.37 , with a spread of 0.32 . These numbers are consistent with the values indicated by Inoue (2011). We notice that the power-law spectral slope is similar to that of both blazars, $2.40 \pm 0.02$ (Abdo et al. 2010e) and to the diffuse $\gamma$-ray background, $2.41 \pm 0.05$ (Abdo et al. 2010d).

The $\gamma$-ray luminosity between energies $\epsilon_{1}$ and $\epsilon_{2}$ is given by

$$
L_{\gamma}\left(\epsilon_{1}, \epsilon_{2}\right)=4 \pi d_{L}^{2}(z) \frac{S_{\gamma}\left(\epsilon_{1}, \epsilon_{2}\right)}{(1+z)^{2-\Gamma}},
$$

where $d_{L}(z)$ is the luminosity distance at the redshift $z$ and $S\left(\epsilon_{1}, \epsilon_{2}\right)$ is the observed energy flux between $\epsilon_{1}$ and $\epsilon_{2}$. The factor $(1+z)^{2-\Gamma}$ is the so-called $K$-correction term that takes into account the redshift modification between the emitted and observed energies. The energy flux, $S_{\gamma}\left(\epsilon_{1}, \epsilon_{2}\right)$, is linked to the photon flux, $F_{\gamma}=\int_{\epsilon_{1}}^{\epsilon_{2}} d \epsilon d N / d \epsilon$ (in units of photons $\mathrm{cm}^{-2} \mathrm{~s}^{-1}$ ), by the relation

$$
S_{\gamma}\left(\epsilon_{1}, \epsilon_{2}\right)=\int_{\epsilon_{1}}^{\epsilon_{2}} \epsilon \frac{d N}{d \epsilon} d \epsilon,
$$

where $d N / d \epsilon$ is the $\gamma$-ray spectrum of the source.

Spectra for the sources in Table 1 have been taken from the 2FGL. They are simple power-law or log-parabola spectra:

$$
\frac{d N}{d \epsilon}=K\left(\frac{\epsilon}{\epsilon_{\text {Pivot }}}\right)^{-\Gamma-\beta \log \left(\epsilon / \epsilon_{\text {Pivot }}\right)},
$$

where $K$ is a normalization factor and the parameter $\beta$ is zero for a power-law spectrum. ${ }^{8}$ Throughout the paper, $\epsilon_{1}=0.1 \mathrm{GeV}$ and $\epsilon_{2}=100 \mathrm{GeV}$, while $\epsilon_{\text {Pivot }}$ has been varied for each source except when dealing with average properties $\left(\epsilon_{\text {Pivot }}=0.1 \mathrm{GeV}\right)$.

Radio luminosity is calculated for a fixed frequency following

$$
L_{r}(v)=\frac{4 \pi d_{L}^{2}(z)}{(1+z)^{1-\alpha_{r}}} S_{r}(v)
$$

where $\alpha_{r}$ is the radio spectral index $\left(\alpha_{\text {core }}\right.$ or $\left.\alpha_{\text {tot }}\right), \Gamma=\alpha_{r}+1$, and $S_{r}(\nu)$ is the radio energy flux at a given frequency.

In Figure 1, we plot the core radio and $\gamma$-ray luminosities for the first 12 MAGNs listed in Table 1 (the last three have been excluded from the sample as explained above). The possible classification into FRI and FRII RGs is also displayed. We have calculated luminosities according to Equations (1)-(4), propagating errors on $\Gamma$ while neglecting errors on the redshift, given their negligible effect. The correlation between $L_{r \text {,core }}$ and $L_{\gamma}$ for the 12 objects is described by the function

$$
\log \left(L_{\gamma}\right)=2.00 \pm 0.98+(1.008 \pm 0.025) \log \left(L_{r, \mathrm{core}}^{5 \mathrm{GHz}}\right),
$$

represented by the solid line in Figure 1, while the relevant $1 \sigma$ error band is shown as a shaded area. The error band is obtained from the errors on both the $\gamma$-ray and radio luminosities, but the

\footnotetext{
7 Making use of Equation (13), we can estimate a core radio luminosity for IC 310 and PKS 0943-76 which is in agreement with Equation (5).

8 Indeed for all the sources considered in our analysis, $\beta=0$, as indicated in the 1FGL and 2FGL catalogs. The only source better reproduced by a log-parabola is $3 \mathrm{C} 84$, which is not included in our analysis.
}

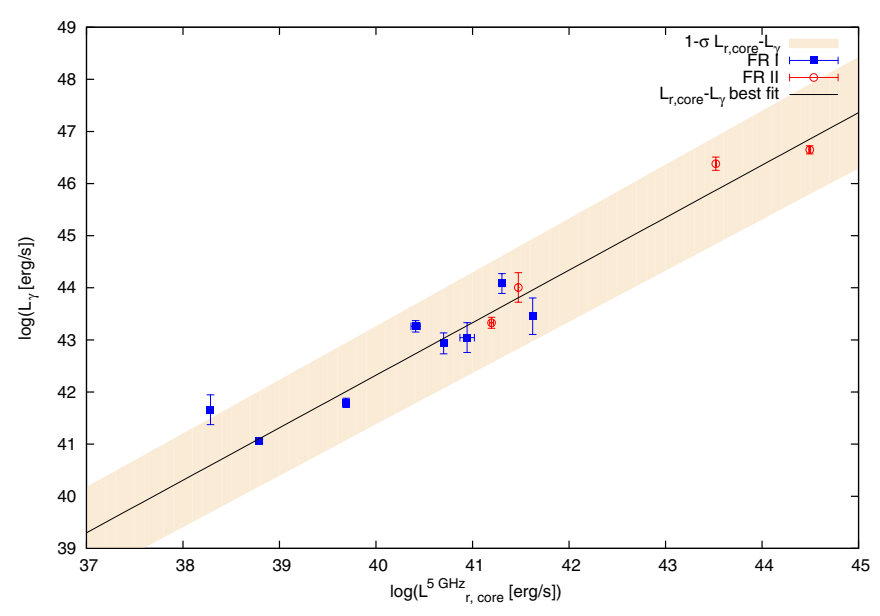

Figure 1. Observed $\gamma$-ray luminosity vs. radio core luminosity at $5 \mathrm{GHz}$ for the MAGNs of Table 1. Blue squares (red open circles) correspond to possible FRI (FRII) classifications. The solid black line represents the calculated correlation as in Equation (5). The light pink shaded area takes into account the $1 \sigma$ error band in the derived correlation function.

(A color version of this figure is available in the online journal.)

former dominates the uncertainty. Equation (5) describes a linear correlation in the log plane with a coefficient very close to one. The $\gamma$-ray luminosities are greater than the radio luminosities by about two orders of magnitude. The uncertainty band of the $\gamma$-ray fluxes measured by the Fermi-LAT spans one order of magnitude around the best fit. The significance of the correlation is tested in Section 4.

It is useful to compare the $L_{r \text {,core }}-L_{\gamma}$ correlation obtained by removing the three sources with non-standard properties from the set of 12 MAGNs in Table 1 . The radio morphology of $3 \mathrm{C}$ 380 shows a clear core-jet structure when observed by VLBI, while at lower resolution it is surrounded by a steep-spectrum low-surface brightness halo (Abdo et al. 2010c; Wilkinson et al. 1991). The FRII 3C 207 behaves as a steep-spectrum radio quasar in the optical band (Abdo et al. 2010c), while PKS 0625-35 has no clear association. We have therefore also calculated the correlation function excluding the galaxies $3 \mathrm{C}$ 380, 3C 207, and PKS 0625-35:

$$
\log \left(L_{\gamma}\right)=2.1 \pm 2.1+(1.005 \pm 0.055) \log \left(L_{r, \text { core }}^{5 \mathrm{GHz}}\right) .
$$

The result is not very different from Equation (5), if it were not for the increased spread in the fitted coefficients. Indeed, 3C 207 and PKS 0625-35 have large errors and 3C 380 is quite close to the correlation with the whole sample.

We also report here the correlation between the total radio luminosity at $5 \mathrm{GHz}$ and the $\gamma$-ray luminosity for the whole sample of 12 sources. The correlation is found to be

$$
\log \left(L_{\gamma}\right)=-2.5 \pm 1.1+(1.095 \pm 0.026) \log \left(L_{r, \text { tot }}^{5 \mathrm{GHz}}\right) .
$$

The experimental values for the total radio luminosity are quoted in Table 1. The fit for the sample of nine sources results in

$$
\log \left(L_{\gamma}\right)=3.5 \pm 2.3+(0.948 \pm 0.056) \log \left(L_{r, \text { tot }}^{5 \mathrm{GHz}}\right) .
$$

The correlation implied by Equation (5) is close to the one obtained by Ghisellini et al. (2005) for the very small sample of three EGRET $\gamma$-ray-loud FRI galaxies (moreover, one of the three galaxies is Cen $\mathrm{A}$, which might have a non-negligible lobe contribution). In the case of blazars, the slopes of the 
correlations between $L_{\gamma}(>100 \mathrm{MeV})$ and the radio luminosity at different frequencies were found to be: $1.07 \pm 0.05$ at $20 \mathrm{GHz}$ (Ghirlanda et al. 2010), $1.2 \pm 0.1$ at $5 \mathrm{GHz}$ (Stecker et al. 1993), and $1.06 \pm 0.02$ at $8.4 \mathrm{GHz}$ (Zeng et al. 2012). The slope coefficient of the correlation for RGs is therefore similar to the correlation for blazars. This might indicate that the $\gamma$-ray emission mechanism is similar for MAGNs and blazars. We therefore assume that the correlation in Equation (5) is a good representation of the luminosity of the cores of MAGNs; we will employ it in the remainder of this work to derive the emission of the MAGN population not detected by the Fermi-LAT but potentially providing a non-negligible diffuse flux.

\section{UPPER LIMITS FROM RADIO-LOUD FRI AND FRII GALAXIES NOT DETECTED BY FERMI-LAT}

In order to test the robustness of the core radio- $\gamma$-ray correlation found in Equation (5), we studied a sample of radioloud FRI and FRII galaxies that have not been detected by Fermi-LAT. For these objects we derived 95\% CL $\gamma$-ray ULs and verified that they are consistent with Equation (5), given the uncertainty band shown in Figure 1. The sample has been extracted from RGs in Kataoka et al. (2011) and Ghisellini et al. (2005) (first and second block in Table 2, respectively) and represents the sources with the highest radio core fluxes at $5 \mathrm{GHz}$. Further selection criteria have been applied in defining the sample for our purposes. From the sample of broad-line RGs whose ULs have been presented in Kataoka et al. (2011), we have excluded Pictor A (Brown \& Adams 2012), detected in the meanwhile, and the sources that do not show a clear FRI or FRII radio morphology classification (RGB J1722+246 and PKS 2251+11 being Seyfert galaxies, and S5 2116+81 being a flat spectrum radio source with a radio jet morphology). Moreover, sources with latitudes below $10^{\circ}$ have been rejected in order to avoid a strong contamination from the Galactic plane foreground; this criterion applies to $4 \mathrm{C} 50.55(b=0.39)$. The same criteria have been applied to sources in Ghisellini et al. (2005), leading to the exclusion of 3C 84, 3C 274, 3C 78 (already detected in $\gamma$ rays), and $3 \mathrm{C} 75$ (which has an atypical $\mathrm{RG}$ morphology). Finally, 3C 317 has been excluded because of its variability (Venturi et al. 2004). Four FRII RGs from the 3CRR catalogue (3C 245, 3C 109, 3C 212, and DA 240) have been added to the sample in order to cover a wider range in radio luminosity (last block in Table 2). Our sample is therefore composed of 17 FRII and 22 FRI RGs.

We have computed $\gamma$-ray flux ULs for the listed galaxies by using the Fermi-LAT Science Tools. ${ }^{9}$ The data-taking period for the analysis is from the starting time of the mission, 2008 August 4, until 2012 September 9. The mission elapsed time interval runs from $239,557,414$ to $368,928,003$. Data have been extracted from a region of interest (ROI) of radius $8^{\circ}$ centered at the position of the source. This radius represents the best angular region for source analysis as long as sources are far from the Galactic plane (Abdo et al. 2009c), and indeed we neglected in this analysis sources that lie below $10^{\circ}$ in latitude. We selected $\gamma$-rays in the energy range $100 \mathrm{MeV}-100 \mathrm{GeV}$.

We are using P7SOURCE_V6 photons. Good survey data were selected according to software recommendations, with the rocking angle selected to be less than $52^{\circ}$. Data selection and preparation eliminated photons from the Earth limb by applying

\footnotetext{
9 http://fermi.gsfc.nasa.gov/ssc/data/analysis/documentation, software version v9r27p1, Instrumental Response Functions P7_V6
}

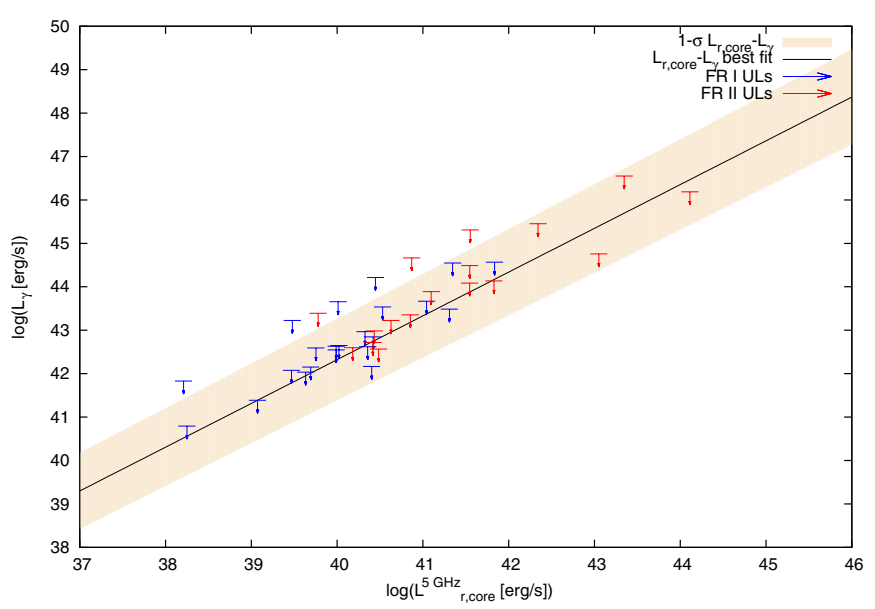

Figure 2. Upper limits on several Fermi-LAT undetected radio-loud MAGNs. Blue (red) symbols refer to possible FRI (FRII) classification. The correlation in Equation (5) (solid black line) is displayed together with the $1 \sigma$ error band (light pink shaded area).

(A color version of this figure is available in the online journal.)

a cut on the zenith angle of $100^{\circ}$. An unbinned maximumlikelihood analysis was performed. In the cases where the fit did not converge, we have performed a binned analysis as recommended. We therefore analyzed the source region with both methods and drew the ULs with the help of the LATAnalysisScripts, ${ }^{10}$ which make use of the UpperLimits.py module.

Each galaxy in the sample was modeled as a point-like source with a power-law spectrum of index $\Gamma=2.5$. This value has been chosen as a nominal spectral index for all MAGNs, similar to Kataoka et al. (2011). We have verified that choosing $\Gamma=2.3$ (closer to the distribution of the spectral indices from Table 1 ) changes the limits by $\sim 10 \%$, while an index of 2.7 leaves the results unchanged. The number of expected counts in the ROI was derived by considering the emission from all sources in the $2 \mathrm{FGL}^{11}$ inside a source region (distance from the target region) of $13^{\circ}\left(8^{\circ}+5^{\circ}\right)$. The fitting procedure leaves the spectral parameters of all the sources inside the ROI free, whereas sources in the region $8^{\circ}<r<13^{\circ}$ have spectral parameters fixed to the values of the 2 FGL. Additional backgrounds are the Galactic diffuse emission and the isotropic diffuse model, which includes the true IGRB and the residual particle contamination. ${ }^{12}$ The diffuse models used in the analysis are: gal_2yearp7v6_v0.fits for the Galactic diffuse model and iso_p7v6source.txt for the isotropic spectral template. All the relevant normalizations have been left as free parameters during the fitting procedure. The method used to compute the ULs is a standard profile likelihood. A 95\% UL was computed when the test statistic (TS) was less than 25. In Table 2, the flux ULs are quoted together with the TS value for both unbinned and binned analyses. Given the systematic uncertainty arising from the different statistical methods, we adopt as a UL the highest value for the flux bound. These conservative ULs are shown in Figure 2 with the luminosity correlation from Equation (5) overlaid. It is evident that the calculated ULs do not fall below the uncertainty band, thus corroborating our core radio and $\gamma$-ray correlation.

\footnotetext{
10 User contributions http://fermi.gsfc.nasa.gov/ssc/data/analysis/user/

11 http://fermi.gsfc.nasa.gov/ssc/data/access/lat/2yr_catalog/

12 http://fermi.gsfc.nasa.gov/ssc/data/access/lat/BackgroundModels.html
} 
Table 2

Flux Upper Limits on a Sample of MAGNs

\begin{tabular}{|c|c|c|c|c|c|c|c|}
\hline MAGN (FRI, FRII) & $z$ & $S_{5 \mathrm{GHz}}^{\text {core }}(\mathrm{Jy})$ & $\mathrm{TS}_{\text {unbinned }}$ & $\mathrm{F}_{\text {unbinned }}^{\mathrm{UL}}$ & $\mathrm{TS}_{\text {binned }}$ & $\mathrm{F}_{\text {binned }}^{\mathrm{UL}}$ & $L_{r, \text { core }}^{5 \mathrm{GHz}}$ \\
\hline $3 \mathrm{C} 18$ (II) & 0.188 & $0.083^{1}$ & $<1$ & 2.7 & 2.6 & 6.0 & $3.51 \times 10^{41}$ \\
\hline B3 0309+411B (II) & 0.134 & $0.320^{2}$ & $\cdots$ & $\cdots$ & $<1$ & 5.8 & $6.73 \times 10^{41}$ \\
\hline 3C 215 (II) & 0.412 & $0.0164^{3}$ & $<1$ & 3.1 & 4.1 & 6.0 & $3.56 \times 10^{41}$ \\
\hline 3C 227 (II) & 0.086 & $0.032^{1}$ & $<1$ & 0.1 & $<1$ & 1.1 & $2.70 \times 10^{40}$ \\
\hline 3C 303 (II) & 0.141 & $0.150^{3}$ & $<1$ & 2.8 & 3.3 & 4.6 & $3.50 \times 10^{41}$ \\
\hline $3 \mathrm{C} 382$ (II) & 0.058 & $0.188^{3}$ & $<1$ & 4.1 & 1.2 & 5.9 & $7.12 \times 10^{40}$ \\
\hline 3C 390.3 (II) & 0.056 & $0.120^{4}$ & $<1$ & 1.7 & 3.0 & 4.7 & $4.26 \times 10^{40}$ \\
\hline 3C 411(II) & 0.467 & $0.078^{5}$ & $\cdots$ & $\cdots$ & $<1$ & 6.1 & $2.2 \times 10^{42}$ \\
\hline $4 \mathrm{C} 74.26$ (II) & 0.104 & $0.100^{6}$ & 1.1 & 5.4 & $<1$ & 5.7 & $1.25 \times 10^{41}$ \\
\hline PKS 2153-69 (II) & 0.028 & $0.300^{7}$ & 4.2 & 6.6 & $<1$ & 6.2 & $2.67 \times 10^{40}$ \\
\hline $3 \mathrm{C} 445$ (II) & 0.056 & $0.086^{1}$ & $<1$ & 0.8 & $<1$ & 1.0 & $3.06 \times 10^{40}$ \\
\hline $3 \mathrm{C} 465$ (I) & 0.029 & $0.270^{3}$ & $\cdots$ & $\cdots$ & $<1$ & 0.5 & $2.5 \times 10^{40}$ \\
\hline 3 C 346 (I) & 0.162 & $0.220^{3}$ & 4.5 & 6.4 & 10.8 & 10.2 & $1.39 \times 10^{39}$ \\
\hline $3 \mathrm{C} 264$ (I) & 0.021 & $0.200^{3}$ & 9.0 & 5.7 & 14.0 & 7.5 & $9.58 \times 10^{39}$ \\
\hline $3 \mathrm{C} 66 \mathrm{~B}(\mathrm{I})$ & 0.022 & $0.182^{3}$ & $\cdots$ & $\cdots$ & $<1$ & 8.3 & $9.31 \times 10^{39}$ \\
\hline 3C 272.1 (I) & 0.003 & $0.180^{3}$ & 5.2 & 5.6 & 5.3 & 6.8 & $1.66 \times 10^{38}$ \\
\hline 3C 315 (I) & 0.1083 & $0.150^{3}$ & $\cdots$ & $\cdots$ & $<1$ & 2.1 & $2.04 \times 10^{41}$ \\
\hline $3 \mathrm{C} 338$ (I) & 0.030 & $0.105^{3}$ & $\cdots$ & $\cdots$ & $<1$ & 4.6 & $1.07 \times 10^{40}$ \\
\hline 3C 293 (I) & 0.045 & $0.100^{1}$ & $<1$ & 1.5 & $<1$ & 1.8 & $2.29 \times 10^{40}$ \\
\hline $3 \mathrm{C} 29$ (I) & 0.045 & $0.093^{3}$ & $<1$ & 1.5 & $<1$ & 4.1 & $2.11 \times 10^{40}$ \\
\hline $3 \mathrm{C} 31$ (I) & 0.017 & $0.092^{3}$ & $\ldots$ & $\ldots$ & $<1$ & 4.0 & $2.83 \times 10^{39}$ \\
\hline $3 \mathrm{C} 310(\mathrm{I})$ & 0.054 & $0.080^{3}$ & $<1$ & 1.2 & $<1$ & 2.1 & $2.63 \times 10^{40}$ \\
\hline 3 C 296 (I) & 0.024 & $0.077^{3}$ & $<1$ & 1.5 & $<1$ & 2.3 & $4.79 \times 10^{39}$ \\
\hline $3 \mathrm{C} 89$ (I) & 0.1386 & $0.049^{3}$ & $\cdots$ & $\cdots$ & $<1$ & 1.8 & $1.10 \times 10^{41}$ \\
\hline $3 \mathrm{C} 449$ (I) & 0.017 & $0.037^{3}$ & $<1$ & 0.5 & $<1$ & 0.8 & $1.19 \times 10^{39}$ \\
\hline $3 \mathrm{C} 288$ (I) & 0.246 & $0.030^{3}$ & $<1$ & 1.5 & 1.6 & 3.7 & $2.22 \times 10^{41}$ \\
\hline $3 \mathrm{C} 305$ (I) & 0.0414 & $0.0295^{3}$ & $\ldots$ & $\ldots$ & $<1$ & 2.1 & $5.66 \times 10^{39}$ \\
\hline $3 \mathrm{C} 83.1 \mathrm{~B}(\mathrm{I})$ & 0.026 & $0.040^{3}$ & 10.0 & 19.7 & 16.5 & 23.2 & $2.89 \times 10^{39}$ \\
\hline $3 \mathrm{C} 424$ (I) & 0.1270 & $0.0180^{3}$ & $\ldots$ & $\ldots$ & $<1$ & 1.6 & $3.39 \times 10^{40}$ \\
\hline $3 \mathrm{C} 438$ (II) & 0.290 & $0.0071^{3}$ & $<1$ & 0.9 & $<1$ & 3.2 & $7.40 \times 10^{40}$ \\
\hline 3 C 386 (I) & 0.018 & $0.120^{3}$ & $\cdots$ & $\cdots$ & $<1$ & 3.2 & $4.15 \times 10^{39}$ \\
\hline 3C 277.3 (I) & 0.0857 & $0.0122^{3}$ & $\cdots$ & $\cdots$ & 4.2 & 5.1 & $1.03 \times 10^{40}$ \\
\hline 3 C 348 (I) & 0.1540 & $0.010^{3}$ & $\ldots$ & $\ldots$ & $<1$ & 5.1 & $2.80 \times 10^{40}$ \\
\hline 3C 433 (II) & 0.102 & $0.005^{3}$ & $\cdots$ & $\cdots$ & $<1$ & 1.9 & $5.96 \times 10^{39}$ \\
\hline $3 \mathrm{C} 442 \mathrm{~A}$ (I) & 0.027 & $0.002^{3}$ & $<1$ & 0.7 & $<1$ & 0.9 & $1.62 \times 10^{38}$ \\
\hline $3 \mathrm{C} 245$ (II) & 1.029 & $0.910^{3}$ & $<1$ & 2.0 & $<1$ & 4.0 & $1.30 \times 10^{44}$ \\
\hline 3C 109 (II) & 0.306 & $0.263^{3}$ & $<1$ & 1.4 & $<1$ & 3.5 & $3.06 \times 10^{42}$ \\
\hline $3 \mathrm{C} 212$ (II) & 1.049 & $0.150^{3}$ & 6.4 & 7.1 & 10.11 & 8.8 & $2.22 \times 10^{43}$ \\
\hline da 240 (II) & 0.036 & $0.105^{3}$ & $<1$ & 1.5 & $<1$ & 2.8 & $1.48 \times 10^{40}$ \\
\hline
\end{tabular}

Notes. Column 1: name of the MAGN (radio classification: FRI or FRII), 2: redshift, 3: measured radio core flux at $5 \mathrm{GHz}$ (Jy), 4: TS of unbinned analysis, 5: 95\% CL upper limit from unbinned analysis on the flux above $100 \mathrm{MeV}$ in units of $10^{-9}$ photons cm $\mathrm{cm}^{-2} \mathrm{~s}^{-1}$, 6: TS of binned analysis, 7: $95 \% \mathrm{CL}$ upper limit from binned analysis on the flux above $100 \mathrm{MeV}$ in units of $10^{-9}$ photons cm $\mathrm{s}^{-2} \mathrm{~s}^{-1}$; 8 : radio core luminosity at $5 \mathrm{GHz}$ in units of $\mathrm{erg} \mathrm{s}^{-1}$.

References. (1) Morganti et al. (1993); (2) Henstock et al. (1995); (3) Third Cambridge Catalogue of Radio Sources; (4) Dodson et al. (2008); (5) Neff et al. (1995); (6) Pearson et al. (1992); (7) Tingay et al. (2002). 


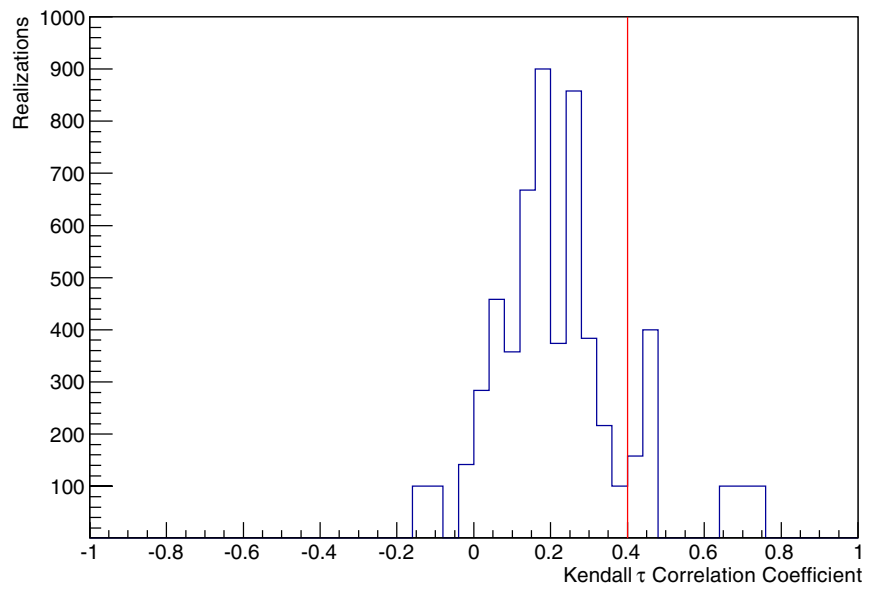

Figure 3. Null hypothesis distribution of $\tau$ correlation coefficients assuming independence between the $\gamma$ and radio wavebands. The null hypothesis distributions are generated from 5800 permutations of $\gamma$-ray luminosities among the galaxies by requiring that the resultant $\gamma$-ray fluxes exceed the flux threshold of $7.7 \times 10^{-9}$ photons $\mathrm{cm}^{-2} \mathrm{~s}^{-1}$. The mean value is 0.223 with a standard deviation of the distribution of $\mathrm{rms}=0.173$. The correlation coefficient of the actual data is represented by the red solid line, $\tau=0.397$.

(A color version of this figure is available in the online journal.)

\section{TEST OF THE RADIO- $\gamma$ CORRELATION}

The correlation established in Equation (5) could be biased by distance dependence of the luminosity- and flux-limited samples (Padovani 1992; Ghirlanda et al. 2011; Inoue 2011). We have tested the strength of the correlation via a partial correlation analysis, in order to verify that the radio-core- $\gamma$-ray luminosity correlation for MAGNs is not spurious.

First, we calculated the Spearman rank-order correlation coefficient. The Spearman correlation coefficients are 0.94, 0.92, and 0.98 between $\log \left(L_{r, \text { core }}^{5 \mathrm{GHz}}\right)$ and $\log \left(L_{\gamma}\right), \log \left(L_{r, \text { core }}^{5 \mathrm{GHz}}\right)$ and redshift, and $\log \left(L_{\gamma}\right)$ and redshift, respectively. The partial correlation coefficient turns out to be 0.51 and the null hypothesis that the two luminosities are uncorrelated is rejected at the 95\% CL.

Moreover, we tested the significance of the radio- $\gamma$-ray correlation by using the modified Kendall $\tau$ rank correlation test proposed by Akritas \& Siebert (1996), which is suitable for partially censored data sets. By following the procedure highlighted in Ackermann et al. (2012a), we performed a Monte Carlo simulation in order to compute the distribution of the $\tau$ correlation coefficients obtained under the null hypothesis of independence between the two wavebands. Starting from the detected sample of 12 sources, we built several data set realizations by scrambling the derived $\gamma$-ray luminosities among galaxies. For each galaxy we then computed the corresponding flux and retained only galaxies with a flux above the minimal $\gamma$-ray flux of the detected sample $\left(7.7 \times 10^{-9}\right.$ photons $\left.\mathrm{cm}^{-2} \mathrm{~s}^{-1}\right)$. If the scrambled sample had fewer than 12 sources above the flux threshold, we randomly extracted an additional source from the UL data set (from Table 2) until the flux threshold was reached. For each scrambled data set, we then computed the Kendall coefficient and we built its distribution as shown in Figure 3. The displayed distribution refers to 5800 realizations of scrambled samples and the red line represents the value of the $\tau$ correlation coefficient of the actual data, $\tau=0.397$.

Finally, we compared the $\tau$ correlation coefficient of the actual data to the distribution of $\tau$ and found that the integral of the distribution above $\tau=0.397$ is 0.05 . This is the probability of obtaining the actual correlation by chance, i.e., the $p$-value of the correlation (the smaller the $p$-value, the greater the probability for the observed correlation being true). As in the case of the Spearman test, we can exclude the correlation happening by chance at the $95 \% \mathrm{CL}$. The result indicates a physical correlation between the core radio emission and the $\gamma$-ray flux of the FermiLAT detected MAGNs.

\section{THE $\gamma$-RAY LUMINOSITY FUNCTION}

The luminosity function for a given energy defines the number of sources emitting at that energy per unit comoving volume, per unit (base 10) logarithm of luminosity:

$$
\rho(L, z)=\frac{d^{2} N}{d \log (L) d V} .
$$

In the radio band, data are available for hundreds of radio-loud MAGNs, depending on the frequency of the survey. Usually radio observations refer to the total emission of the AGN, including the central region, jets, and radio lobes. Only for a limited number of objects detected at low radio frequencies (around 0.1 to a few $\mathrm{GHz}$ ) has the flux from the central core alone been measured. The RLF is derived phenomenologically by fitting data on the emission of the radio sample. Results on the total RLF are quite well established (Willott et al. 2001; Dunlop \& Peacock 1990; Yuan \& Wang 2012), while the literature on the core RLF is still limited (Yuan \& Wang 2012), given the scarcity of experimental data.

Unfortunately, deriving the GLF by fitting the $\gamma$-ray measurements is not feasible due to the small size of the $\gamma$-ray loud MAGN sample. Following previous attempts applied to blazars (Stecker \& Salamon 1996; Kazanas \& Perlman 1997; Narumoto \& Totani 2006; Stecker \& Venters 2011) and, to a lesser extent, to RGs (Ghisellini et al. 2005; Inoue 2011), we derived the GLF from the RLF by exploiting the correlation between radio and $\gamma$-ray luminosities found in Section 2. We assume that

$$
N_{\gamma}=k N_{r},
$$

where the normalization, $k$, takes into account our ignorance of the number of radio-loud MAGNs emitting in $\gamma$-rays as well ( $N_{r}$ and $N_{\gamma}$, respectively). From Equation (9), it follows that $N=\int d V \int \rho(L, z) d \log L$ and therefore the GLF is defined through an RLF by

$$
\rho_{\gamma}\left(L_{\gamma}, z\right)=k \rho_{r}\left(L_{r}, z\right) \frac{d \log L_{r}}{d \log L_{\gamma}} .
$$

Given the results of the previous sections, the above equation takes the specific form

$$
\rho_{\gamma}\left(L_{\gamma}, z\right)=k \rho_{r, \text { core }}\left(L_{r, \text { core }}^{5 \mathrm{GHz}}\left(L_{\gamma}\right), z\right) \frac{d \log L_{r, \mathrm{core}}^{5 \mathrm{GHz}}\left(L_{\gamma}\right)}{d \log L_{\gamma}},
$$

where $\rho_{r, \text { core }}$ refers to the RLF of the cores of the MAGNs. If our hypothesis of a correlation between the core radio and $\gamma$-ray emission is physical, as supported by the results on the ULs (see previous section), we might expect $k$ values not too far from 1 . In other words, each RG with a bright radio core is expected to emit in the $\gamma$-ray band as well. The correlation between radio and $\gamma$-ray luminosities is assumed to be a specific analytical expression, Equation (5), shown to be in very good agreement with the data. In this sense, the scatter in the correlation 


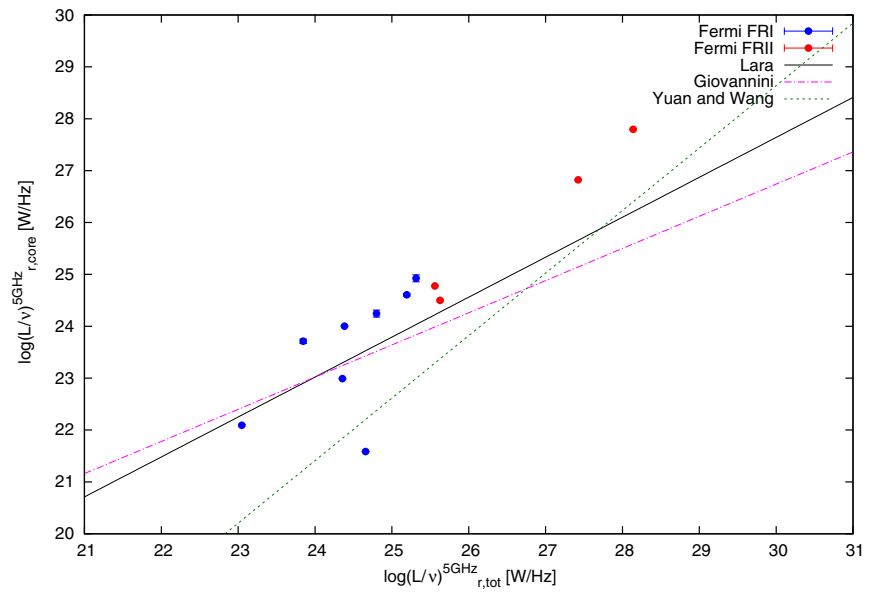

Figure 4. Radio core luminosity vs. total radio luminosity at $5 \mathrm{GHz}$. The solid black line corresponds to Equation (13), while the pink dot-dashed and the green dotted lines correspond to Equations (14) and (15), respectively. Blue squares (red circles) are the experimental data for our sample of FRI (FRII) taken from Table 1. All the points contain error bars, which are indeed very small.

(A color version of this figure is available in the online journal.)

derives only from errors in the experimental data and not in a potential scatter on the luminosity form. The radio luminosity is energetically weaker, according to Equation (5). As already noted, the lack of a reliable core RLF from data prevents us from using Equation (12) directly. The only core RLF in Yuan \& Wang (2012) finds a strong negative evolution, while it is expected that the core and lobes should co-evolve with redshift. RGs, as well as lobes that are detected at low frequency, evolve positively and there is very little evidence for the presence of RGs with a "switched-off" core, as there should be if lobes and cores had a different evolution. Moreover, the same authors reported the positive evolution of RGs and derived a correlation between total radio flux and core flux that would yield a positively-evolving core RLF with a simple transformation of their total RLF (using their correlation). We will therefore make use of the total RLF and obtain the core RLF through the link between total and core radio luminosities.

As a first ingredient, we need a correlation between radio core and total luminosities. In Figure 4, we display the correlation between $L_{v, \text { tot }}^{5 \mathrm{GHz}}$ and $L_{v, \text { core }}^{5 \mathrm{GHz}}$. The three curves correspond to:

$$
\log L_{v, \text { core }}^{5 \mathrm{GHz}}=4.2 \pm 2.1+(0.77 \pm 0.08) \log L_{\nu, \text { tot }}^{1.4 \mathrm{GHz}}
$$

from Lara et al. (2004) (black solid curve),

$$
\log L_{v, \text { core }}^{5 \mathrm{GHz}}=7.6 \pm 1.1+(0.62 \pm 0.04) \log L_{v, \text { tot }}^{408 \mathrm{MHz}}
$$

from Giovannini et al. (2001) (pink dot-dashed curve), and

$$
\log L_{v, \text { tot }}^{408 \mathrm{MHz}}=7.10 \pm 0.90+(0.83 \pm 0.04) \log L_{v, \text { core }}^{5 \mathrm{GHz}}
$$

from (Yuan \& Wang 2012; green dotted curve). We report all the luminosities at $5 \mathrm{GHz}$, assuming a power-law dependence of $L / v \propto v^{-\alpha}$, with $\alpha_{\text {tot }}=0.80$ for the total radio emission (as assumed, e.g., in Inoue 2011). It is clear from Figure 4 that the experimental data for our MAGN sample are best represented by the correlation proposed by Lara et al. (2004). We will therefore adopt Equation (13) throughout the paper. The possible systematics introduced by this correlation are likely compensated, at least to a good extent, by the fit to the cumulative number counts (see the following section).
The core RLF may be obtained from the total one following the same reasoning as for Equations (10) and (11):

$$
\rho_{r, \text { core }}\left(L_{r, \text { core }}, z\right)=\rho_{r, \text { tot }}\left(L_{r, \text { tot }}, z\right) \frac{d \log L_{r, \text { tot }}}{d \log L_{r, \text { core }}},
$$

where we expect that the number of MAGNs showing core and total emission is almost the same. We adopted as the total RLF the one derived in (Willott et al. 2001; Model C with $\Omega_{M}=0$ ) and shifted luminosities from $151 \mathrm{MHz}$ to $5 \mathrm{GHz}$ according to the power law explained above. We converted the comoving volume to the standard $\Lambda \mathrm{CDM}$ cosmology by using the conversion factor

$$
\eta(z)=\frac{d^{2} V_{W} / d z d \Omega}{d^{2} V / d z d \Omega},
$$

where $d^{2} V_{W} / d z d \Omega$ is the comoving volume element used by Willott et al. (2001),

$$
\frac{d^{2} V_{W}}{d z d \Omega}=\frac{c^{3} z^{2}(2+z)^{2}}{4 H_{0, W}^{3}(1+z)^{3}}
$$

$c$ is the speed of light; and $H_{0, W}=50 \mathrm{~km} \mathrm{~s}^{-1} \mathrm{Mpc}^{-1}$. In the cosmological model $\Lambda \mathrm{CDM}$, the comoving volume element is defined as

$$
\begin{aligned}
& \frac{d^{2} V}{d z d \Omega}= \\
& \frac{c d_{L}(z)^{2}}{H_{0}(1+z)^{2} \sqrt{\left(1-\Omega_{\Lambda}-\Omega_{M}\right)(1+z)^{2}+(1+z)^{3} \Omega_{M}+\Omega_{\Lambda}}} .
\end{aligned}
$$

We finally obtained the GLF by inserting Equation (16) into Equation (12):

$$
\begin{array}{r}
\rho_{\gamma}\left(L_{\gamma}, z\right)=k \rho_{r, \text { tot }}\left(L_{r, \text { tot }}^{5 \mathrm{GHz}}\left(L_{r, \text { core }}^{5 \mathrm{GHz}}\left(L_{\gamma}\right)\right), z\right) \\
\cdot \frac{d \log L_{r, \text { core }}^{5 \mathrm{GHz}}}{d \log L_{\gamma}} \frac{d \log L_{r, \text { tot }}^{5 \mathrm{GHz}}}{d \log L_{r, \text { core }}^{5 \mathrm{GHz}}} .
\end{array}
$$

The $d \log L_{r, \text { core }}^{5 \mathrm{GHz}} / d \log L_{\gamma}$ will be computed from Equation (5), while the $d \log L_{r, \text { tot }}^{5 \mathrm{GHz}} / d \log L_{r \text {,core }}^{5 \mathrm{GHz}}$ derives from the total-core correlation, Equation (13).

\section{PREDICTIONS FOR THE SOURCE-COUNT DISTRIBUTION}

An important observable for the correctness of our method is provided by the source-count distribution of MAGNs measured by the Fermi-LAT. The source-count distribution, known also as $\log N-\log S$, is the cumulative number of sources $N\left(>F_{\gamma}\right)$ detected above a threshold flux, $F_{\gamma}$. We have derived the experimental source-count distribution of the 12 MAGNs of our sample following Abdo et al. (2010f):

$$
N\left(>F_{\gamma}\right)=\sum_{i=1}^{N\left(>F_{\gamma, i}\right)} \frac{1}{\omega\left(F_{\gamma, i}\right)}
$$

where the sum runs on all the $i$-sources with a $\gamma$-ray flux $F_{\gamma, i}>$ $F_{\gamma}$, and $\omega\left(F_{\gamma, i}\right)$ is the flux-dependent detection efficiency compatible with our sample. As shown in Abdo et al. (2009b, 2010f), at faint fluxes the Fermi-LAT more easily detects hardspectrum sources rather than sources with a soft spectrum. 
Sources with a photon index of $\Gamma=1.5$ can be detected down to fluxes that are a factor $>20$ fainter than those of a source with a photon index of 3.0. Given this strong selection effect, the intrinsic photon-index distribution is necessarily different from the observed one. This effect is taken into account by the detection efficiency. Since the latter is not available for the MAGN sample, we reasonably assume it is the same as for blazars of the 1FGL and take it from Abdo et al. (2010e). We demonstrate in the Appendix that an empirical estimation of the efficiency for the 2FGL blazars does not change the results of our analysis.

The theoretical source-count distribution, $N_{\mathrm{th}}\left(>F_{\gamma}\right)$, for a $\gamma$-ray flux $F_{\gamma}$ is calculated following the definition of GLF in Equation (9):

$$
\begin{aligned}
N_{\mathrm{th}}\left(>F_{\gamma}\right)= & 4 \pi \int_{\Gamma_{\max }}^{\Gamma_{\min }} \frac{d N}{d \Gamma} d \Gamma \int_{0}^{z_{\max }} \frac{d^{2} V}{d z d \Omega} d z \\
& \times \int_{L_{\gamma}\left(F_{\gamma}, z, \Gamma\right)}^{L_{\gamma}^{\max }} \frac{d L_{\gamma}}{L_{\gamma} \ln (10)} \rho_{\gamma}\left(L_{\gamma}, z, \Gamma\right),
\end{aligned}
$$

where $L_{\gamma}\left(F_{\gamma}, z, \Gamma\right)$ is the $\gamma$-ray luminosity of an RG at redshift $z$ whose photon spectral index is $\Gamma$ and photon flux is $F_{\gamma}$ (integrated above $100 \mathrm{MeV}$ ). The spectral index distribution, $d N / d \Gamma$, is assumed to be Gaussian, which is analogous to blazars (Abdo et al. 2010e). Indeed, any observing instrument with finite sensitivity, and Fermi is no exception to this, inevitably selects sources near its detection threshold, resulting in an asymmetric distribution of observed spectral indices. The detected MAGN spectral-index distribution, similar to the one from blazars, appears as non-Gaussian and asymmetric (more hard sources than soft sources). A proper test, including selection effects, requires a larger sample and is beyond the scope of this paper. On the other hand, there are no indications that support deviations from a standard symmetric Gaussian distribution. Our treatment of the distribution in photon indices does not explicitly correct for errors in individual measurements, which can artificially increase the distribution spread (Venters \& Pavlidou 2007). However, since the errors in individual photonindex measurements are quite small, we expect this effect not to be very important. Its effect might slightly decrease the expected emission at high energies, which would further reduce the importance of any cascade emission for this component of the IGRB, which we do not calculate here. The comoving volume, $d^{2} V /(d z d \Omega)$, is computed according to Equation (19). We fix $\Gamma_{\min }=1.0, \Gamma_{\max }=3.5, z_{\max }=6$, and $L_{\gamma, \max }=10^{50} \mathrm{erg} \mathrm{s}^{-1}$.

Figure 5 shows the theoretical $N_{\text {th }}\left(>F_{\gamma}\right)$, calculated from Equation (22), with several bands of uncertainty, overlaid with the experimental source-count distribution from Equation (21). Their comparison is discussed here only as a consistency check on the validity of the assumptions involved in Equation (22) - in particular, of the ratio of MAGNs emitting in $\gamma$-rays relative to those emitting in the radio core, i.e., the $k$ parameter in Equation (22). The data points for the experimental $N\left(>F_{\gamma}\right)$ are in fact highly correlated, and a fit to those points is not statistically meaningful. Nonetheless, it is useful to fit the theoretical $N_{\text {th }}\left(>F_{\gamma}\right)$ to the experimental source-count distribution to constrain the only free parameter, $k$, in this calculation. Additionally, the shape of the function predicting $N\left(>F_{\gamma}\right)$ is essentially driven by the radio luminosity density function and not by the fit to the experimental source-count distribution.

The black dashed line in Figure 5 has been derived from the best fit parameters of Equation (5) $\left(\log \left(L_{\gamma}\right)=2.00 \pm\right.$

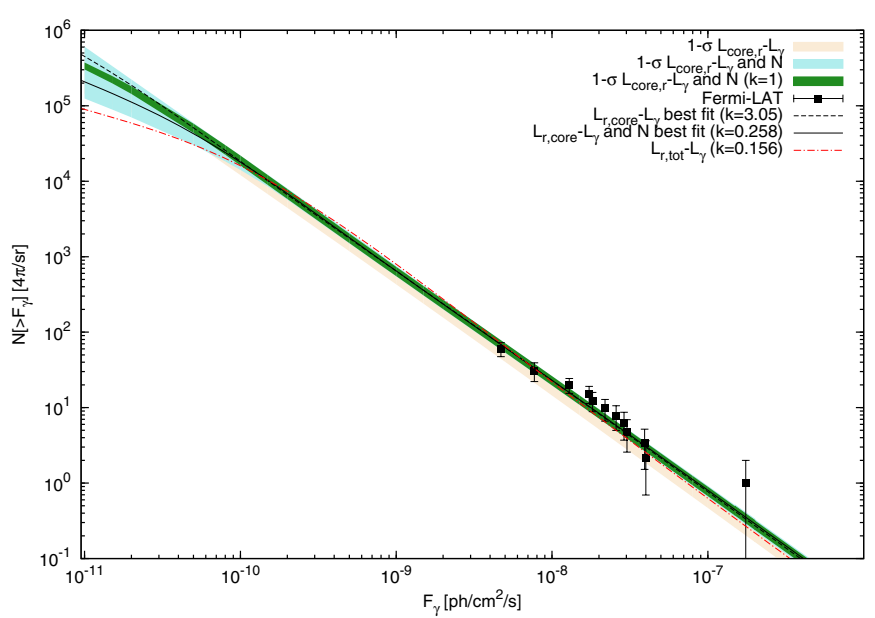

Figure 5. Source-count distribution as a function of the integrated $\gamma$-ray flux. Fermi-LAT data are represented by black squares with $1 \sigma$ error bars. The black dashed line (pink shaded area) shows the source-count distribution predicted with the best fit configuration ( $1 \sigma$ uncertainty band) for the $L_{r \text {, core }}-L_{\gamma}$ correlation $\log \left(L_{\gamma}\right)=2.00 \pm 0.98+(1.008 \pm 0.025) \log \left(L_{r \text {,core }}^{5 \mathrm{GHz}}\right)$. The black solid line (cyan shaded area) corresponds to the source-count distribution predicted after the minimization on the $L_{r \text {,core }}-L_{\gamma}$ best fit ( $1 \sigma$ uncertainty band) correlation and $k$ (see the text for details). The green shaded area includes all the configurations with $k=1$. The red dot-dashed curve has been obtained with the $L_{r, \text { tot }}-L_{\gamma}$ correlation according to Equation (7).

(A color version of this figure is available in the online journal.)

$\left.0.98+(1.008 \pm 0.025) \log \left(L_{r, \text { core }}^{5 \mathrm{GHz}}\right)\right)$, whose fit to the experimental source-count distribution gives $k=3.05 \pm 0.20$ with a $\chi^{2}=$ 6.98 (for 11 degrees of freedom). This indicates that the best fit radio-core- $\gamma$-ray correlation function slightly underpredicts the distribution of MAGNs observed by the Fermi-LAT.

To obtain the bands depicted in Figure 5, we have proceeded as follows:

1. we have calculated the $N\left(>F_{\gamma}\right)$ for all the correlation coefficients falling in the $1 \sigma$ uncertainty band for the $L_{r \text {, core }}-L_{\gamma}$ relationship (Figure 1);

2. for each combination of these coefficients, we have determined $k$ from the comparison with the $\log N-\log S$ (pink shaded area);

3 . the configuration with the lowest $\chi^{2}$ among all the configurations explored at point 2 predicts the best $N\left(>F_{\gamma}\right)$ (black solid line, $k=0.258$ );

4. all the configurations giving a $1 \sigma$ variation from the lowest $\chi^{2}$ (minimal $\left.\chi^{2}+3.53\right)$ span the cyan shaded area.

The red dot-dashed curve was obtained for the radio-total$\gamma$-ray luminosity correlation in Equation (7) and the total RLF in Willott et al. (2001). This hypothesis leads to a lower number of sources at the lowest fluxes. The pink shaded area (and similarly the cyan band) is quite narrow because of the degree of freedom implied by $k$, which is fitted on the experimental $\log N-\log S$ for all the $\gamma$-ray luminosities falling in the $1 \sigma$ band of Equation (5).

Finally, the green shaded band was obtained by fixing the normalization factor, $k$, in Equations (10) and (11) equal to 1 , which represents the ideal situation in which we predict that each MAGN has a radio-loud central region emitting in $\gamma$-rays as well. We have varied the luminosity correlation in Equation (5) within its $1 \sigma$ band. The lowest $\chi^{2}$ is 6.80 (for 10 degrees of freedom), and the green band describes the relevant $1 \sigma$ uncertainty. This result is an important test of the validity of our initial assumption that an MAGN with a radio-core emission also emits photons in the $\gamma$-ray energy band, likely via SSC and 


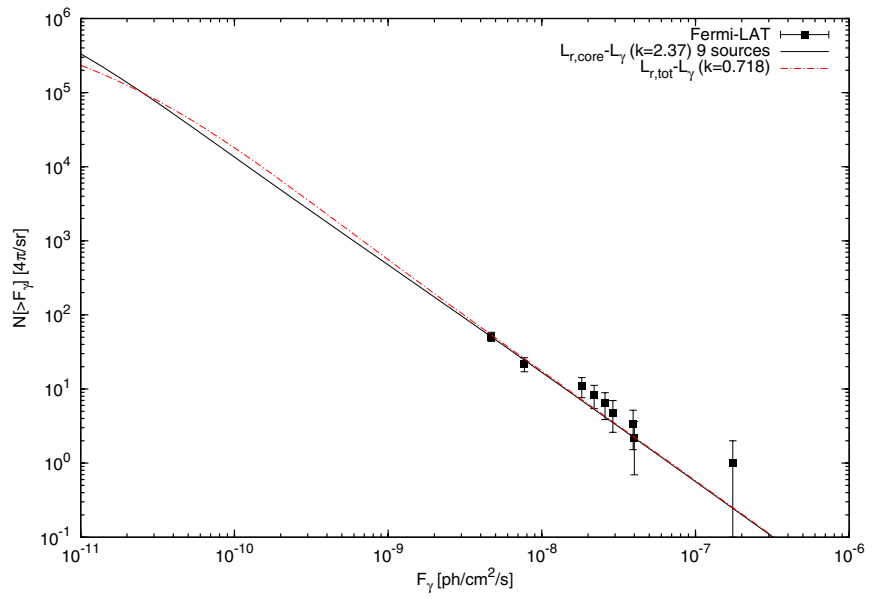

Figure 6. Source-count distribution as a function of the integrated $\gamma$-ray flux for 9 RGs. The Fermi-LAT data are represented by black squares with $1 \sigma$ error bars. The black solid line corresponds to the source-count distribution predicted with the best fit configuration for the $L_{r \text {,core }}-L_{\gamma}$ correlation in Equation (6) and $N\left(>F_{\gamma}\right)$. The red dot-dashed curve has been obtained with the $L_{r \text {,tot }}-L_{\gamma}$ correlation according to Equation (8).

(A color version of this figure is available in the online journal.)

EC processes. It is remarkable that the band is a good fit to the Fermi-LAT data.

Given the uncertain classification of some of the sources, as explained in Section 2, we also provide the source-count distribution for the 9 sources with firm FRI or FRII classifications (Figure 6). We show the experimental and the theoretical source-count distribution predicted when the three galaxies $3 \mathrm{C}$ 380, 3C 207, and PKS 0625-35 are excluded from the analysis. The black solid line is the same as in Figure 5, but obtained with nine data points and employing Equation (6) for the $L_{r \text {,core }}-L_{\gamma}$ luminosity correlation function. The result is compatible with the data, with $\chi^{2}=4.65$ and the normalization for the source number distribution $k=2.37$. The red dot-dashed curve is the same as in Figure 5 but obtained from the total RLF and Equation (8) and minimized with respect to the nine data points.

\section{THE DIFFUSE $\gamma$-RAY EMISSION FROM MAGNs}

The diffuse $\gamma$-ray flux due to the whole population of MAGNs may be estimated as follows:

$$
\begin{aligned}
& \frac{d^{2} F(\epsilon)}{d \epsilon d \Omega}=\int_{\Gamma_{\min }}^{\Gamma_{\max }} d \Gamma \frac{d N}{d \Gamma} \int_{0}^{z_{\max }} \frac{d^{2} V}{d z d \Omega} d z \int_{L_{\gamma, \min }}^{L_{\gamma, \max }} \frac{d F_{\gamma}}{d \epsilon} \\
& \frac{d L_{\gamma}}{L_{\gamma} \ln (10)} \rho_{\gamma}\left(L_{\gamma}, z\right)\left(1-\omega\left(F_{\gamma}\left(L_{\gamma}, z\right)\right)\right) \exp \left(-\tau_{\gamma, \gamma}(\epsilon, z)\right) .
\end{aligned}
$$

The minimum $\gamma$-ray luminosity value is set to $10^{41} \mathrm{erg} \mathrm{s}^{-1}$, the maximum at $10^{50} \mathrm{erg} \mathrm{s}^{-1}$. The term $\omega\left(F_{\gamma}\left(L_{\gamma}, z\right)\right)$ is the detection efficiency of the Fermi-LAT at the photon flux $F_{\gamma}$, which corresponds to the flux from a source with a $\gamma$-ray luminosity $L_{\gamma}$ at redshift $z . d N / d \Gamma$ is the photon spectral index distribution (see Equation (22)). $d F_{\gamma} / d \epsilon$ is the intrinsic photon flux at energy $\epsilon$ for an MAGN with $\gamma$-ray luminosity $L_{\gamma}$ (Venters et al. 2009; Yan et al. 2012):

$$
\frac{d F_{\gamma}}{d \epsilon}=\frac{(1+z)^{2-\Gamma}}{4 \pi d_{L}(z)^{2}} \frac{(2-\Gamma)}{\left[\left(\frac{\epsilon_{2}}{\epsilon_{1}}\right)^{2-\Gamma}-1\right]}\left(\frac{\epsilon}{\epsilon_{1}}\right)^{-\Gamma} \frac{L_{\gamma}}{\epsilon_{1}^{2}} .
$$

High-energy $\gamma$-rays $(\epsilon>20 \mathrm{GeV})$ propagating in the universe are absorbed by the interaction with the extragalactic background light (EBL), cosmic optical radiation, and infrared background (Gould \& Schréder 1966; Jelley 1966; Stecker et al. 1992, 2006; Salamon \& Stecker 1998; Mazin \& Raue 2007; Razzaque et al. 2009; Gilmore et al. 2009; Finke et al. 2010; Ackermann et al. 2012b; Abramowski et al. 2013) with an optical depth $\tau_{\gamma, \gamma}(\epsilon, z)$. In this study, we adopt the attenuation model of Finke et al. (2010). The $\gamma$-ray absorption creates electron-positron pairs, which can scatter off the CMB photons through IC scattering yielding a secondary cascade emission at lower $\gamma$-ray energies. We include the cascade emission from high-energy $\gamma$-rays following Inoue $\&$ Ioka (2012) and Kneiske $\&$ Mannheim (2008) and accounting for the first generation of electrons produced from the interaction of $\gamma$-rays with the EBL. (In the considered energy range the correction for the second generation of electrons is negligible). We assume a maximum $\gamma$-ray energy of $10 \mathrm{TeV}$ as this is the indicative largest energy sampled by current generation TeV telescopes (Wakely \& Horan 2013; Sanchez et al. 2013). At these energies, the interaction with the CMB photons is well described by Thomson scattering.

Within these hypotheses, the cascade emission is computed according to Equation (23), where the intrinsic photon flux, $d F_{\gamma} / d \epsilon$, is replaced by

$$
\frac{d F_{\gamma}^{\mathrm{casc}}}{d \epsilon}(\epsilon, z)=\frac{(1+z)}{4 \pi d_{L}(z)^{2}} \int_{\gamma_{e, \text { min }}}^{\gamma_{e, \max }} \frac{d N_{\gamma_{e} \epsilon}}{d t d \epsilon} \frac{d N_{e}}{d \gamma_{e}} t_{\mathrm{IC}}(z) d \gamma_{e},
$$

where $t_{\mathrm{IC}}(z)$ is the energy-loss time of an electron with a, Lorentz factor $\gamma_{e}$. The term $d N_{\gamma_{e} \epsilon} / d t d \epsilon$ is the IC scattered photon spectrum per unit time:

$$
\frac{d N_{\gamma_{e} \epsilon}}{d t d \epsilon}=\frac{3 \sigma_{T} c}{4 \gamma_{e}^{2}} \int_{0}^{1} \frac{d x}{x} \frac{d n_{\mathrm{CMB}}}{d x}\left(x\left(\xi, \gamma_{e}\right), z\right) f(x),
$$

where $\sigma_{T}$ is the Thomson scattering cross section, $f(x)=$ $2 x \ln x+x+1-2 x^{2}(0<x<1)$, and $x=\epsilon_{\gamma, i} / 4 \gamma_{e}^{2} \xi$. Here, $\epsilon_{\gamma, i}=2 \gamma_{e} m_{e} c^{2}$ is the energy of intrinsic photons and $d n_{\mathrm{CMB}} / d \xi$ is the CMB photon density with energy $\xi$. The integration in Equation (25) runs from $\gamma_{e, \min }=$ $\max \left[\left(E_{\gamma} / \epsilon\right)^{1 / 2} / 2,100 \mathrm{MeV} / 2 m_{e} c^{2}\right]$ to $\gamma_{e, \max }=E_{\max } / 2 m_{e} c^{2}$. The electron spectrum $d N_{e} / d \gamma_{e}$ is given by

$$
\frac{d N_{e}}{d \gamma_{e}}=\frac{d_{L}(z)^{2}}{(1+z)} \frac{d \epsilon_{\gamma, i}}{d \gamma_{e}} \frac{d F_{\gamma}}{d \epsilon}\left(1-\exp \left(-\tau_{\gamma, \gamma}\right)\right),
$$

where $d F_{\gamma} / d \epsilon$ is given by Equation (24).

Figure 7 shows the diffuse $\gamma$-ray flux due to the MAGN population as a function of $\gamma$-ray energy, along with the Fermi-LAT data for the IGRB (Abdo et al. 2010d). The cyan shaded area derives from the $1 \sigma$ uncertainty band on the $L_{r \text {,core }}-L_{\gamma}$ correlation and on the $k$ parameter in the source-count distribution (see description of the cyan shaded area in Figure 5). The upper edge of the uncertainty band skims the IGRB data points, while the lower limit is almost an order of magnitude below the data. The band itself is nearly a factor of 10 wide. The flux integrated above $100 \mathrm{MeV}$ is $5.69 \times 10^{-7} \mathrm{~cm}^{-2} \mathrm{~s}^{-1} \mathrm{sr}^{-1}$ for the lower bound of the uncertainty band and $4.91 \times 10^{-6}$ $\mathrm{cm}^{-2} \mathrm{~s}^{-1} \mathrm{sr}^{-1}$ for the upper one. These values compare with $1.03 \times 10^{-5} \mathrm{~cm}^{-2} \mathrm{~s}^{-1} \mathrm{sr}^{-1}$ derived from the experimental data (Abdo et al. 2010d). The green band has been obtained by fixing $k=1$ as described in Section 6 (Figure 5). It corresponds to the 


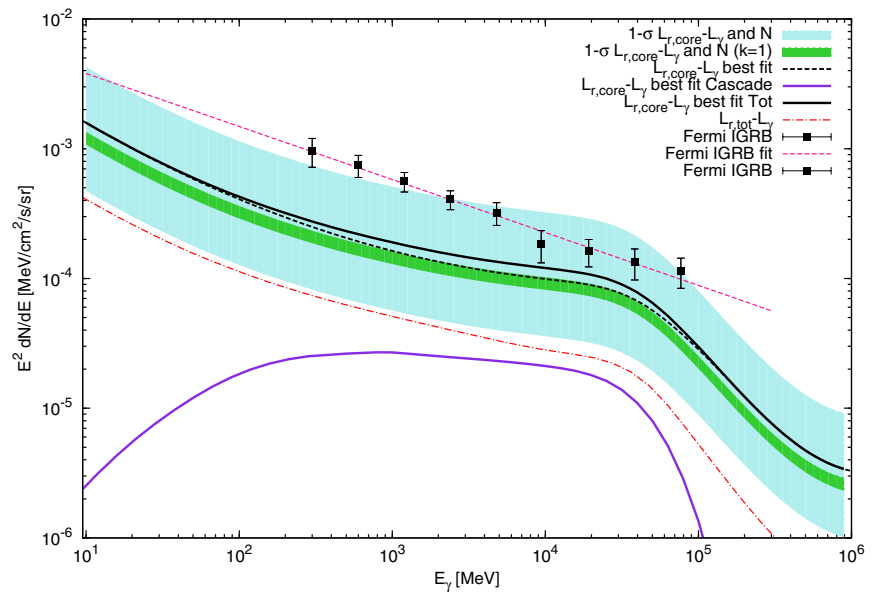

Figure 7. Diffuse $\gamma$-ray flux due to the MAGNs as a function of $\gamma$-ray energy. The black squares correspond to the IGRB measured by the Fermi-LAT (Abdo et al. 2010d) and best-fitted by the magenta dashed curve. The cyan shaded area derives from the $1 \sigma$ uncertainty band on the $L_{r \text {,core }}-L_{\gamma}$ correlation and on the $k$ parameter in the source-count distribution. The solid black line is obtained from the best fit on the $L_{r, \text { core }}-L_{\gamma}$ correlation, while the dashed black line illustrates the relevant flux without the contribution from the cascade emission, depicted by the violet solid line. The green band corresponds to $k=1$ at $1 \sigma \mathrm{CL}$ (see discussion on Figure 5). The red dot-dashed curve shows the diffuse flux obtained when assuming a $L_{r, \text { tot }}-L_{\gamma}$ correlation.

(A color version of this figure is available in the online journal.)

case in which all the MAGNs with a radio-loud central region emit in $\gamma$-rays as well and with a phenomenological model that nicely fits all the experimental constraints.

The flux calculated for the best fit coefficients of the $L_{r, \text { core }}-L_{\gamma}$ correlation and $k=3.05$ (see description of the black dashed line in Figure 5) is displayed in Figure 7. The corresponding cascade emission is illustrated as a violet curve. It shows a flat behavior (with respect to the $E^{2}$ normalization adopted in the figure) between about $200 \mathrm{MeV}$ and $30 \mathrm{GeV}$, while it drops sharply at higher energies. At $100 \mathrm{MeV}, 1 \mathrm{GeV}$, and $10 \mathrm{GeV}$ the cascade emission is about $4.5 \%, 16 \%$, and $21 \%$ (respectively) of the nonabsorbed flux. As a consistency check, we estimated the energy flux of the photons absorbed by interaction with the EBL and compared it to that from the cascade emission, as well as the total energy flux from the photons arriving at the LAT which are not absorbed by EBL (dashed black line in Figure 7). The latter is obtained by integrating Equation (23) multiplied by the energy, between $100 \mathrm{MeV}$ and $10 \mathrm{TeV}$, and has a value of $2.35 \times 10^{-3} \mathrm{MeV} \mathrm{cm}^{-2} \mathrm{sr}^{-1} \mathrm{~s}^{-1}$. By simply replacing the EBL attenuation term $\exp \left(-\tau_{\gamma, \gamma}(\epsilon, z)\right)$ with its complement $\left(1-\exp \left(-\tau_{\gamma, \gamma}(\epsilon, z)\right)\right)$, and performing the same integral, we computed the energy flux of those photons that get absorbed by the EBL and can be reprocessed through the cascade, obtaining a value of $2.96 \times 10^{-4} \mathrm{MeV} \mathrm{cm}^{-2} \mathrm{sr}^{-1} \mathrm{~s}^{-1}$. This can be considered as an UL to the cascade emission and is, in fact, slightly higher than its actual flux of $1.93 \times 10^{-4} \mathrm{MeV} \mathrm{cm}^{-2} \mathrm{sr}^{-1} \mathrm{~s}^{-1}$, which represents only $8 \%$ of the total MAGN flux.

Our predictions are for an MAGN population whose $\gamma$-ray emission is assumed to originate from the central region of the active galaxy and modeled from the core RLF. The dotdashed red line represents the flux derived when the $\gamma$-ray luminosity is correlated with the total radio luminosity according to Equation (7), and the total RLF (Willott et al. 2001; see description of the red dot-dashed line in Figure 5). The effect of EBL absorption is clear from the softening of the flux above $50 \mathrm{GeV}$. The deviation from a pure power-law shape below $\sim 30 \mathrm{GeV}$ is due to integration over the photon-index distribution. We note that the contribution of unresolved blazars (Abdo et al. 2010e) has a very similar slope but is lower than the one obtained for MAGNs in this paper. The two uncertainty bands nearly touch each other. Inoue (2011) reported that MAGNs can contribute to the IGRB at the level of $10 \%-63 \%$, which is a range compatible with our result.

The flux displayed in Figure 7 results from an integration up to a maximum luminosity of $10^{50} \mathrm{erg} \mathrm{s}^{-1}$. The result does not depend on the maximal luminosity of integration, confirming that the photons come from very numerous and very faint sources. A confirmation of the negligible contribution of bright sources to the overall flux is that the flux at $1 \mathrm{GeV}$ (multiplied by $E^{2}$ ) for the 15 galaxies of Table 1 is $3.5 \times 10^{-6} \mathrm{MeV} \mathrm{cm}^{-2} \mathrm{~s}^{-1}$ $\mathrm{sr}^{-1}$, more than two orders of magnitude below our estimated diffuse flux. We finally observe that shifting the lower luminosity from $10^{41} \mathrm{erg} \mathrm{s}^{-1}$ down to $10^{38} \mathrm{erg} \mathrm{s}^{-1}$ would lead to a $15 \%$ greater isotropic intensity.

Our predictions may be compared to the results reported by Inoue (2011). In that paper, the flux from unresolved MAGNs has been obtained for a single model, which is contained in our uncertainty band and shows a different shape with respect to our representative cases (solid and dashed lines in Figure 7). Possible differences between the two procedures are probably due to the fact that Inoue (2011) works within a smaller and different RG sample. We both establish a $L_{r \text {,core }}-L_{\gamma}$ correlation. However, we convert the total RLF by Willott et al. (2001) to a core RLF, whereas Inoue (2011) does not make this transformation. Data on the source-number distribution are different, in particular the Fermi-LAT data points at the lowest fluxes. A final possible difference might reside in a different angular conversion factor in the RLF coefficients in Willott et al. (2001).

\section{CONCLUSIONS}

We have calculated the diffuse $\gamma$-ray emission from the population of MAGNs at all redshifts.

We first established the existence (at 95\% CL) of a correlation between the radio-core $\left(L_{r, \text { core }}\right)$ and the $\gamma$-ray $\left(L_{\gamma}\right)$ luminosities of the MAGNs detected by the Fermi-LAT. This correlation is substantially linear in the log plane, the radio luminosity being two orders of magnitude lower than the $\gamma$-ray luminosity. Extensive tests showed that this correlation is not likely to be a spurious effect due to the source distance. We also calculated the ULs on the $\gamma$-ray emission from 33 radio-loud MAGNs undetected by the Fermi-LAT and showed that these are compatible with the core-radio- $\gamma$-ray luminosity correlation within $1 \sigma$ errors.

We then used this correlation to infer a GLF from a well established RLF and further tested the former against the sourcecount distribution measured by the Fermi-LAT. We correctly predicted the number of detected $\gamma$-ray sources, with values of the normalization factor $k$ between the population of MAGNs emitting in radio and $\gamma$-rays that are close to one. Even when constraining $k=1$, our GLF matched the Fermi-LAT source-count distribution, nicely confirming the robustness and simplicity of the luminosity correlation we derived.

Using our GLF, and after taking into account $\gamma$-ray absorption from a model of EBL, we predicted the diffuse $\gamma$-ray flux due to MAGNs between $10 \mathrm{MeV}$ and $1 \mathrm{TeV}$. We found an intensity of about $2 \times 10^{-4} \mathrm{MeV} \mathrm{cm}^{-2} \mathrm{~s}^{-1} \mathrm{sr}^{-1}$ at $1 \mathrm{GeV}$, embedded in a uncertainty band of nearly a factor of ten. At all Fermi-LAT energies, the best fit MAGN contribution 
is $20 \%-30 \%$ of the measured IGRB flux. The lower edge of the uncertainty band is about one order of magnitude smaller than the IGRB data while the upper edge skims the data below a few $\mathrm{GeV}$ and slightly overestimates them from a few $\mathrm{GeV}$ to around $50 \mathrm{GeV}$. Our uncertainty band includes the results found by Inoue (2011), based on a correlation between $\gamma$-rays and the total radio luminosity. At higher energies, the flux softens because of the EBL absorption. The intensity from MAGNs integrated above $100 \mathrm{MeV}$ is $9.83 \times 10^{-7}, 2.61 \times 10^{-6}$, and $8.56 \times 10^{-6}$ photons $\mathrm{cm}^{-2} \mathrm{~s}^{-1} \mathrm{sr}^{-1}$, when considering the lower, best-fit, and upper curve of the band reported in Figure 7. These numbers represent $9.5 \%, 25 \%$, and $83 \%$ of the IGRB, respectively. The analogous calculation for the two blazar populations of BL Lacs and FSRQs gives $7.83_{-2.34}^{+1.09} \times$ $10^{-7}$ photons $\mathrm{cm}^{-2} \mathrm{~s}^{-1} \mathrm{sr}^{-1}$ (about $8 \%$ of the IGRB) for the former (Abdo et al. 2010e) and $9.66_{-1.09}^{+1.67} 10^{-7}$ photons $\mathrm{cm}^{-2} \mathrm{~s}^{-1}$ $\mathrm{sr}^{-1}$ (about $10 \%$ of the IGRB) for the latter (Ajello et al. 2012). The integrated flux for star-forming galaxies (Ackermann et al. 2012a) is instead 8.19-7.31 $\times 10^{-7}$ photons $\mathrm{cm}^{-2} \mathrm{~s}^{-1} \mathrm{sr}^{-1}$, which contributes $4.1 \%$ (14.8\%) of the IGRB at minimum (maximum) and about $8 \%$ at its best fit value.

In conclusion, we have calculated the diffuse $\gamma$-ray flux from unresolved MAGNs. The main original results of our analysis include (1) the derivation of a $\gamma$-ray-radio core luminosity correlation for the MAGNs observed by the Fermi-LAT; (2) the test of this correlation against ULs from tens of radio loud MAGNs undetected in $\gamma$-rays; (3) tests of the correlation in order to verify that the radio-core- $\gamma$-ray luminosity correlation for MAGNs is not spurious; (4) the calculation of the GLF from the core radio one; (5) evaluation of the uncertainties affecting $\gamma$-ray flux predicted from the unresolved MAGN population. We have found that the cosmological population of faint and numerous MAGNs gives a sizable diffuse extragalactic flux that - when added to the contribution from other sources, e.g., blazars (Abdo et al. 2010e); star-forming galaxies (Ackermann et al. 2012a); millisecond pulsars (Faucher-Giguere \& Loeb 2010; Siegal-Gaskins et al. 2011); cascade from ultra-highenergy cosmic rays (Ahlers et al. 2010); radio-quiet AGNs (Inoue \& Totani 2009); large scale structures (Gabici \& Blasi 2003); strong galactic foreground (Keshet et al. 2004); and cosmic-ray interaction in the solar system (Moskalenko \& Porter 2009) — could entirely explain the observed IGRB. This scenario would leave very little room for more exotic sources, such as dark matter in the halo of our Galaxy (Calore et al. 2013).

M.D.M. and F.D. warmly acknowledge S. Massaglia for invaluable insights in the preliminary stage of this paper and P.D. Serpico for useful comments. M.A. acknowledges support from grant NNH09ZDA001N for the study of the origin of the Isotropic Gamma-Ray Background. F.C. acknowledges support from the German Research Foundation (DFG) through grant BR 3954/1-1.

The Fermi LAT Collaboration acknowledges generous ongoing sup- port from a number of agencies and institutes that have supported both the development and the operation of the LAT as well as scientific data analysis. These include the National Aeronautics and Space Administration and the Department of Energy in the United States; the Commissariat à l'Energie Atomique and the Centre National de la Recherche Scientifique/ Institut National de Physique Nucléaire et de Physique des Particules in France; the Agenzia Spaziale Italiana and the Istituto Nazionale di Fisica Nucleare in Italy; the Ministry of Education, Culture, Sports, Science and Technology (MEXT), High
Energy Accelerator Re- search Organization (KEK), and Japan Aerospace Exploration Agency (JAXA) in Japan; and the K. A. Wallenberg Foundation, the Swedish Research Council, and the Swedish National Space Board in Sweden. Additional support for science analysis during the operations phase is gratefully acknowledged from the Istituto Nazionale di Astrofisica in Italy and the Centre National d'Etudes Spatiales in France.

\section{APPENDIX}

\section{ESTIMATION OF THE 2FGL EFFICIENCY}

Out of the 12 MAGNs considered in our analysis, 8 galaxies (3C 78, 3C 274, Cen A, NGC 6251, PKS 0625-35, 3C 111, 3C 207, and 3C 380) are found in the 1FGL, 8 (3C 274, Cen A, NGC 6251, Cen B, Fornax A, PKS 0625-35, 3C 207, and 3C 380) are in the 2FGL, while 3C 120 is listed in Abdo et al. (2010c) and Pictor A has been revealed in Brown \& Adams (2012). The efficiency employed in our analysis is taken from Abdo et al. (2010e), which refers to the 1FGL blazar catalog. Since the source detection efficiency was not published for the 2FGL, we have assumed in this paper that the same 1FGL efficiency holds for all the MAGN in Table 1. In this section, we infer an efficiency for the 2FGL catalog and check if the $\log N-\log S$ associated to the $2 \mathrm{FGL}$ sources is consistent with the results discussed in Section 6.

We start from the blazar $\log N-\log S$ count distribution established in Abdo et al. (2010e). As a first step, we search all the 2FGL blazars with TS $>25$ (in accordance with the MAGN TS) and $|b|>10^{\circ}$ (in order to exclude the contamination from the galactic plane). For all the selected sources, we compute the flux $F_{\gamma}$ from $100 \mathrm{MeV}$ up to $100 \mathrm{GeV}$ according to Equations (2) and (3). We have considered a flux range from $10^{-9}$ to $10^{-5}$ photons $\mathrm{cm}^{-2} \mathrm{~s}^{-1}$ divided in to $N$ bins. The efficiency $\omega\left(F_{\gamma}^{k}\right)$ at a flux $F_{\gamma}^{k} \in\left(F_{\gamma}^{k, \min }, F_{\gamma}^{k, \max }\right)(k=\{1 \ldots, N\})$ may be estimated as

$$
\omega\left(F_{\gamma}^{k}\right)=(1+\eta) \frac{N_{\text {blazars }}^{k}}{\Delta \Omega \int_{F_{\gamma}^{k, \text { min }}}^{F_{\text {,max }}} \frac{d N}{d F_{\gamma}} d F_{\gamma}},
$$

where $\Delta \Omega$ is the solid angle associated with $|b|>10^{\circ}$ and $N_{\text {blazars }}^{k}$ is the number of selected blazars with flux $F_{\gamma} \in$ $\left(F_{\gamma}^{k, \text { min }}, F_{\gamma}^{k, \max }\right)$. The integrand $d N / d F_{\gamma}$ is the flux distribution of blazars, and the term $\int_{F_{\gamma}^{k, \text { min }}}^{F^{k, \text { max }}}\left(d N / d F_{\gamma}\right) d F_{\gamma}$ in Equation (A1) represents the expected number of blazars. The incompleteness of the 2FGL catalog, $\eta$, is given by the ratio of unassociated sources to the total number of sources. In the 2FGL (for TS > 25 and $|b|>10^{\circ}$ ), there are 1042 sources out of which 169 are unassociated, giving $\eta \approx 0.16$. The flux distribution $d N / d F_{\gamma}$ of the 2FGL being unknown, we assume it to be the one from the 1FGL taken from (Abdo et al. 2010e; for TS $>50$ and $\left.|b|>20^{\circ}\right)$.

In Figure 8, we show the estimated efficiency found with the method described here. The error bars represent the uncertainties on $d N / d F_{\gamma}$ and the Poisson errors associated with the observed number of blazars, $N_{\text {blazars }}^{k}$, counted in each flux bin. We also overlap the 1FGL efficiency (Abdo et al. 2010e). The two efficiencies are similar at high fluxes $\left(F_{\gamma}>4 \times 10^{-8}\right.$ photons $\mathrm{cm}^{-2} \mathrm{~s}^{-1}$ ), while at lower values the 2FGL efficiency is shifted to the left side. This is due to the selection criteria used for deriving the efficiency, which are looser for 2FGL (TS > 25 and $|b|>10^{\circ}$ ) with respect to the 1FGL (TS $>50$ and $|b|>20^{\circ}$ ). 


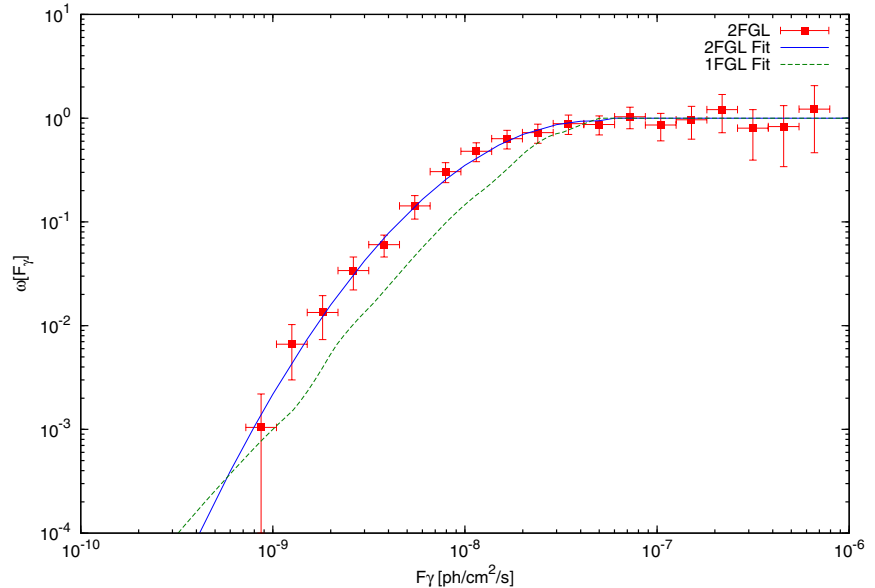

Figure 8. Evaluated efficiency for the 2FGL (red points fitted by the blue curve) along with the 1FGL efficiency (Abdo et al. 2010e; green dashed curve), as a function of the integrated $\gamma$-ray flux $F_{\gamma}$.

(A color version of this figure is available in the online journal.)

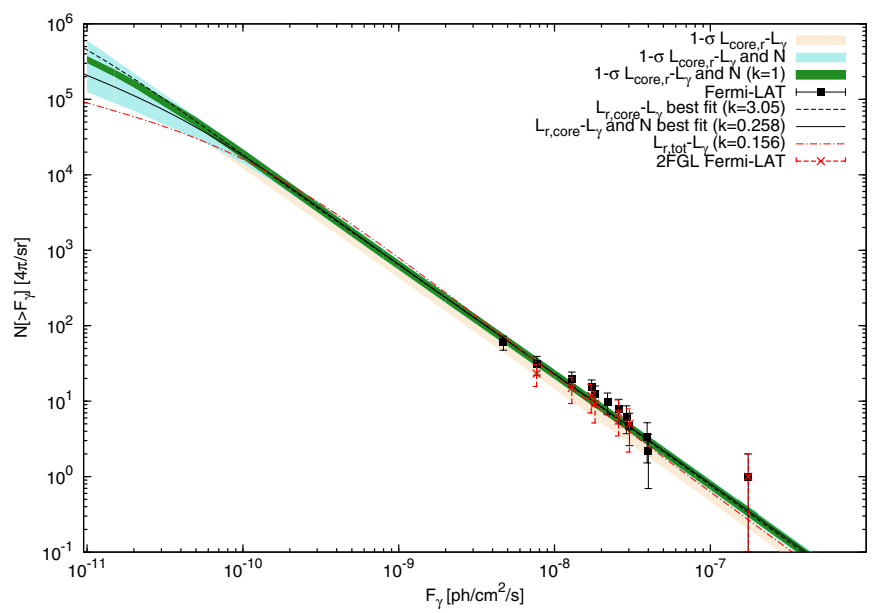

Figure 9. Same as in Figure 5, with the addition of the red points indicating the number count evaluated with the efficiency estimated for the $2 \mathrm{GL}$ (as in Figure 8), for the eight MAGNs detected in the 2FGL catalog.

(A color version of this figure is available in the online journal.)

Finally, in Figure 9 we display the $\log N-\log S$ as in Figure 5 (for 12 MAGNs) to which we add the (red) points corresponding to the number count computed for the eight MAGNs in the 2FGL catalog and assuming the detection efficiency estimated for the 2FGL as in Figure 8. We can conclude that the source-number count for the 2FGL sample, and with newly estimated efficiency, is compatible with the results obtained for the whole MAGN population treated with the 1FGL efficiency. Additionally, a slightly different normalization can be safely compensated for by the free normalization parameter, $k$, (see discussion in Section 6) and will not change the flux prediction derived in Section 7.

\section{REFERENCES}

Abazajian, K. N., Blanchet, S., \& Harding, J. P. 2011, PhRvD, 84, 103007 Abdo, A., Ackermann, M., Ajello, M., et al. 2009a, ApJ, 707, 55 Abdo, A., Ackermann, M., Ajello, M., et al. 2010a, ApJ, 719, 1433 Abdo, A. A., Ackermann, M., Ajello, M., et al. 2009b, ApJ, 700, 597 Abdo, A. A., Ackermann, M., Ajello, M., et al. 2009c, ApJ, 699, 31 Abdo, A. A., Ackermann, M., Ajello, M., et al. 2010b, Sci, 328, 725 Abdo, A. A., Ackermann, M., Ajello, M., et al. 2010c, ApJ, 720, 912 Abdo, A. A., Ackermann, M., Ajello, M., et al. 2010d, PhRvL, 104, 101101 Abdo, A. A., Ackermann, M., Ajello, M., et al. 2010e, ApJ, 720, 435
Abdo, A. A., Ackermann, M., Ajello, M., et al. 2010f, ApJ, 715, 429 Abramowski, A., Acero, F., Aharonian, F., et al. 2013, A\&A, 550, A4 Ackermann, M., Ajello, M., Allafort, A., et al. 2011a, ApJ, 741, 30 Ackermann, M., Ajello, M., Allafort, A., et al. 2011b, ApJ, 743, 171 Ackermann, M., Ajello, M., Allafort, A., et al. 2012a, ApJ, 755, 164 Ackermann, M., Ajello, M., Allafort, A., et al. 2012b, Sci, 338, 1190 Ahlers, M., Anchordoqui, L. A., Gonzalez-Garcia, M. C., Halzen, F., \& Sarkar, S. 2010, APh, 34, 106

Ajello, M., Shaw, M. S., Romani, R. W., et al. 2012, ApJ, 751, 108

Akritas, M. G., \& Siebert, J. 1996, MNRAS, 278, 919

Barthel, P. D. 1989, ApJ, 336, 606

Becker, R. H., White, R. L., \& Edwards, A. L. 1991, ApJS, 75, 1

Bhattacharya, D., Sreekumar, P., \& Mukherjee, R. 2009, RAA, 9, 1205

Brown, A. M., \& Adams, J. 2012, MNRAS, 421, 2303

Burgess, A. M., \& Hunstead, R. W. 2006, ApJ, 131, 114

Burns, J. O., Feigelson, E. D., \& Schreier, E. J. 1983, ApJ, 273, 128

Calore, F., Di Mauro, M., \& Donato, F. 2013, PhRvD, submitted (arXiv:1303.3284)

Dermer, C. D., \& Schlickeiser, R. 1993, ApJ, 416, 458

Dodson, R., Fomalont, E. B., Wiik, K., et al. 2008, ApJS, 175, 314

Dondi, L., \& Ghisellini, G. 1995, MNRAS, 273, 583

Dunlop, J. S., \& Peacock, J. A. 1990, MNRAS, 247, 19

Ekers, R. D., Wall, J. V., Shaver, P. A., et al. 1989, MNRAS, 236, 737

Evans, D. A., Hardcastle, M. J., Croston, J. H., Worrall, D. M., \& Birkinshaw, M. 2005, MNRAS, 359, 363

Fanaroff, B. L., \& Riley, J. M. 1974, MNRAS, 167, 31P

Faucher-Giguere, C.-A., \& Loeb, A. 2010, JCAP, 01, 005

Finke, J. D., Razzaque, S., \& Dermer, C. D. 2010, ApJ, 712, 238

Gabici, S., \& Blasi, P. 2003, APh, 19, 679

Geldzahler, B. J., \& Fomalont, E. B. 1984, AJ, 89, 1650

Ghirlanda, G., Ghisellini, G., Tavecchio, F., \& Foschini, L. 2010, MNRAS, 407, 791

Ghirlanda, G., Ghisellini, G., Tavecchio, F., Foschini, L., \& Bonnoli, G. 2011, MNRAS, 413, 852

Ghisellini, G., Tavecchio, F., \& Chiaberge, M. 2005, A\&A, 432, 401

Gilmore, R. C., Madau, P., Primack, J. R., Somerville, R. S., \& Haardt, F. 2009, MNRAS, 399, 1694

Giovannini, G., Cotton, W. D., Feretti, L., Lara, L., \& Venturi, T. 2001, ApJ, 552,508

Giovannini, G., Feretti, L., Gregorini, L., \& Parma, P. 1988, A\&A, 199, 73

Gould, R. J., \& Schréder, G. 1966, PhRvL, 16, 252

Grandi, P. 2012, IJMPS, 8, 25

Grandi, P., \& Torresi, E. on behalf of the FERMI-LAT Collaboration. 2012a, arXiv: 1205.1686

Grandi, P., Torresi, E., \& Stanghellini, C. 2012b, ApJL, 751, L3

Gregory, P. C., \& Condon, J. J. 1991, ApJS, 75, 1011

Henstock, D. R., Browne, I. W. A., Wilkinson, P. N., et al. 1995, ApJS, 100, 1 Inoue, Y. 2011, ApJ, 733, 66

Inoue, Y., \& Ioka, K. 2012, PhRvD, 86, 023003

Inoue, Y., \& Totani, T. 2009, ApJ, 702, 523

Israel, F. P., Raban, D., Booth, R. S., \& Rantakyrö, F. T. 2008, A\&A, 483, 741 Jelley, J. V. 1966, PhRvL, 16, 479

Jones, P. A., Lloyd, B. D., \& McAdam, W. B. 2001, MNRAS, 325, 817

Kadler, M., Eisenacher, D., Ros, E., et al. 2012, A\&A, 538, L1

Kataoka, J., Stawarz, Ł., Takahashi, Y., et al. 2011, ApJ, 740, 29

Katsuta, J., Tanaka, Y. T., Stawarz, Ł., et al. 2013, A\&A, 550, A66

Kazanas, D., \& Perlman, E. 1997, ApJ, 476, 7

Keshet, U., Waxman, E., \& Loeb, A. 2004, JCAP, 04, 006

Kneiske, T. M., \& Mannheim, K. 2008, A\&A, 479, 41

Kuehr, H., Witzel, A., Pauliny-Toth, I. I. K., \& Nauber, U. 1981, A\&AS, 45,367

Kusenko, A., \& Voloshin, M. B. 2012, PhLB, 707, 255

Laing, R. A., Riley, J. M., \& Longair, M. S. 1983, MNRAS, 204, 151

Lara, L., Giovannini, G., Cotton, W. D., et al. 2004, A\&A, 421, 899

Linfield, R., \& Perley, R. 1984, ApJ, 279, 60

Mantovani, F., Mack, K.-H., Montenegro-Montes, F. M., Rossetti, A., \& Kraus, A. 2009, A\&A, 502, 61

Maraschi, L., Ghisellini, G., \& Celotti, A. 1992, ApJL, 397, L5

Massardi, M., Ekers, R. D., Murphy, T., et al. 2008, MNRAS, 384, 775

Massaro, F., \& Ajello, M. 2011, ApJL, 729, L12

Mazin, D., \& Raue, M. 2007, A\&A, 471, 439

Morganti, R., Killeen, N. E. B., \& Tadhunter, C. N. 1993, MNRAS, 263, 1023

Moskalenko, I. V., \& Porter, T. A. 2009, ApJL, 692, L54

Mullin, L. M., Hardcastle, M. J., \& Riley, J. M. 2006, MNRAS, 372, 113

Nagar, N. M., Wilson, A. S., \& Falcke, H. 2001, ApJL, 559, L87

Narumoto, T., \& Totani, T. 2006, ApJ, 643, 81

Neff, S. G., Roberts, L., \& Hutchings, J. B. 1995, ApJS, 99, 349 
Nolan, P. L., Abdo, A. A., Ackermann, M., et al. 2012a, ApJS, 199, 31 Nolan, P. L., Abdo, A. A., Ackermann, M., et al. 2012b, yCat, 219, 90031 Padovani, P. 1992, A\&A, 256, 399

Padovani, P., Ghisellini, G., Fabian, A. C., \& Celotti, A. 1993, MNRAS, 260, L21

Pauliny-Toth, I. I. K., Kellermann, K. I., Davis, M. M., Fomalont, E. B., \& Shaffer, D. B. 1972, AJ, 77, 265

Pearson, T. J., Blundell, K. M., Riley, J. M., \& Warner, P. J. 1992, MNRAS, 259, 13P

Perley, R. A., Roser, H.-J., \& Meisenheimer, K. 1997, A\&A, 328, 12

Razzaque, S., Dermer, C. D., \& Finke, J. D. 2009, ApJ, 697, 483

Salamon, M. H., \& Stecker, F. W. 1994, ApJL, 430, L21

Salamon, M. H., \& Stecker, F. W. 1998, ApJ, 493, 547

Sanchez, D. A., Fegan, S., \& Giebels, B. 2013, A\&A, 554, A75

Siegal-Gaskins, J. M., Reesman, R., Pavlidou, V., Profumo, S., \& Walker, T. P. 2011, MNRAS, 415, 1074S

Spinrad, H., Marr, J., Aguilar, L., \& Djorgovski, S. 1985, PASP, 97, 932

Stawarz, Ł., Kneiske, T. M., \& Kataoka, J. 2006, ApJ, 637, 693

Stecker, F. W., de Jager, O. C., \& Salamon, M. H. 1992, ApJL, 390, L49
Stecker, F. W., Malkan, M. A., \& Scully, S. T. 2006, ApJ, 648, 774

Stecker, F. W., \& Salamon, M. H. 1996, ApJ, 464, 600

Stecker, F. W., Salamon, M. H., \& Malkan, M. A. 1993, ApJL, 410, L71

Stecker, F. W., \& Venters, T. M. 2011, ApJ, 736, 40

Tingay, S. J., Reynolds, J. E., Tzioumis, A. K., et al. 2002, ApJS, 141, 311

Urry, C. M., \& Padovani, P. 1995, PASP, 107, 803

Venters, T. M., \& Pavlidou, V. 2007, ApJ, 666, 128

Venters, T. M., Pavlidou, V., \& Reyes, L. C. 2009, ApJ, 703, 1939

Venturi, T., Dallacasa, D., \& Stefanachi, F. 2004, A\&A, 422, 515

Wakely, S., \& Horan, D. 2013, http://tevcat.uchicago.edu

Wilkinson, P. N., Akujor, C. E., Cornwell, T. J., \& Saikia, D. J. 1991, MNRAS, 248,86

Willott, C. J., Rawlings, S., Blundell, K. M., Lacy, M., \& Eales, S. A. 2001, MNRAS, 322, 536

Wright, A. E., Griffith, M. R., Burke, B. F., \& Ekers, R. D. 1994, ApJS, 91, 111

Yan, D., Zeng, H., \& Zhang, L. 2012, MNRAS, 422, 1779

Yuan, Z., \& Wang, J. 2012, ApJ, 744, 84

Zeng, H. D., Yan, D. H., Sun, Y. Q., \& Zhang, L. 2012, ApJ, 749, 151

Zhou, M., \& Wang, J. 2013, ApJ, 769, 153 